\title{
10 Epik um einen Fluss: Geographie, Geschichte und Mittellatinistik als Schlüssel zur Sachsenepik
}

\begin{abstract}
Résumé : Le contenu géographique et historique des chansons de geste ayant pour sujet les guerres saxonnes de Charlemagne (essentiellement les Saisnes et, comme représentants de textes français perdus, les branches I et V de la Karlamagnús-Saga norroise) est de nos jours encore largement sous-estimé. À l'aide des deux Vitae de la reine Mathilde, l'article fait remonter jusqu'au $X^{\mathrm{e}}$ siècle le thème légendaire du duel victorieux de Charlemagne contre Guitechin, scène cruciale des Saisnes ; il fait ressortir la base géographique du rôle primordial des fleuves dans toutes ces œuvres et l'origine historique du motif non moins important de la construction d'un pont par Charlemagne ; il explique tant les différences importantes des trois versions principales (pont sur le Rhin à Wesel dans la KMS I, sur le Rhin à Worms dans la KMS V, sur la Ruhr près de Dortmund dans les Saisnes) que la place marquante de Dortmund dans chacune des trois. Enfin, il identifie comme noyau historique des Saisnes la prise d'assaut en 775 de la Hohensyburg, qui domine, au sud de Dortmund, la vallée de la Ruhr du sommet d'une berge escarpée de plus de $100 \mathrm{~m}$ de hauteur. Pourtant, dans la francophonie ce noyau narratif fut au moins ravivé et précisé par les marchands wallons qui, fréquentant régulièrement Dortmund au moins depuis le $\mathrm{XI}^{\mathrm{e}}$ siècle, devaient chaque fois gagner la hauteur de la Hohensyburg. Au passage, l'article élucide un passage controversé du Liber Historiae Francorum, l'homonymie des deux fleuves sépiques` du nom de Rune (la Ruhr, l'Arga de Pampelune) et la genèse du lat. méd. Tremonia 〈Dortmund».
\end{abstract}

\section{Die Texte}

\section{a Überblick}

Vergleichend untersuchen lassen sich die epischen Darstellungen von Karls Sachsenkrieg nur, wenn man zunächst den Textbestand betrachtet.

Die wichtigste Darstellung liegt in afrz. Sprache selbst vor, Bodels Chanson des Saisnes (um 1200, im Folgenden kurz Saisnes). ${ }^{1}$

1 Im Folgenden zitiert nach Jehan Bodel, La Chanson des Saisnes, ed. Annette Brasseur, 2 Bde., Genève 1989. - Zwei weitere afrz. Epen, Doon de Mayence (Mitte 13. Jh., vielleicht Remaniement eines älteren Liedes) und Gaufrey (noch 13. Jh.), könnte man nur in einem ganz äußerlichen Sinn des Wortes zur «Sachsenepik» rechnen. Da in ihnen außer dem Grundfaktum, dass Karl gegen Sachsen kämpft, nichts der historischen und fast nichts der geographischen Realität entspricht, behandle ich sie nicht (bis auf ein unten in Anm. 51 erwähntes Detail). Auch die Aufnahme des Sachsenkriegs-Stoffes in Sammelwerke wie die von Girart d’Amiens (um

Anmerkung: Erstmals veröffentlicht in: Mittellateinisches Jahrbuch 51 (2016), 221-258.

Ә Open Access. (c) 2019 Gustav Adolf Beckmann, publiziert von De Gruyter. (c))BY-NC-ND Dieses Werk ist lizenziert unter der Creative Commons Attribution-NonCommercial-NoDerivatives 4.0 Lizenz.

https://doi.org/10.1515/9783110615692-010 
Nur in altnord. Übertragung fassbar sind zwei Darstellungen in der Karlamagnús saga (13. Jh., kurz: KMS), zum einen in Kap. 46-47 der Ed. Unger (1860, entsprechend Kap. A43-A44 der Ed. Loth, 1980) der ersten Branche der KMS (kurz: KMS I $46 \mathrm{f}$.), ${ }^{2}$ zum andern in deren gesamter fünfter Branche (KMS V), die nur in der Ed. Unger vorliegt. Beide Texte wollen wie die Saisnes Erzählungen des ganzen Sachsenkrieges sein; das macht sie strukturell beurteilbar und mit den Saisnes vergleichbar.

Der lat. Pseudo-Turpin (um 1140-1150, kurz PT) berührt Karls Sachsenkrieg nur in seinem Kap. 33, das inhaltlich ein Nachtrag, aber schon im Codex Calixtinus enthalten ist. Während der Belagerung von Nobles (hier allerdings in Grenoble umgedeutet) wird Roland von Karl, den die Sachsen in einer Burg bei Warmacia $\left\langle\right.$ Worms ${ }^{3}$ eingeschlossen haben, zur schnellen Hilfe herbeizitiert. Gott lässt wie bei Jericho die Mauern von Grenoble von selbst fallen, und Roland trifft früh genug zur Befreiung seines Onkels ein. $\mathrm{Zu}$ fragen ist aus unserer jetzigen Perspektive nur, wie Worms in den Sachsenkrieg kommt. Doch findet sich die Worms-Szene in einen breiteren Erzählkontext eingebettet auch in der KMS V; somit lässt sich die Frage von dort aus besser beantworten.

Im Span. gehört zu den Materialien frz.-epischer Herkunft, die im ältesten Ms. (Madrid B. N. 1187, spätes 13. Jh.) der Gran Conquista de Ultramar noch fehlen, ${ }^{4}$ aber im 14. Jh. in die Kompilation aufgenommen wurden, das nur wenige Zeilen umfassende Résumé eines Sachsenkrieges, der, soweit man das bei dieser Kürze sagen kann, inhaltlich zwischen der KMS V und Bodel steht. ${ }^{5}$ Zudem existieren Romanzen, die zwar inhaltlich mit den Saisnes zusammenhängen, deren Genese aber so starke Eigenprobleme aufwirft, ${ }^{6}$ dass sie für das Studium der Saisnes unergiebig sind.

1300), Jean d’Outremeuse (spätes 14. Jh.) und David Aubert (1458) kann hier nicht untersucht werden.

2 Karlamagnús saga. Branches I, III, VII et IX, Édition bilingue projetée par Knud Togeby et Pierre Halleux. Texte norrois édité par Agnete Loth. Traduction française par Annette [Patron-] Godefroit. Avec une étude par Povl Skårup, København 1980. - Karlamagnus saga ok kappa hans, ed. Carl Richard Unger, Kristiania [Oslo] 1860. Wie schon Daniel W. Lacroix in seiner frz. Übersetzung La Saga de Charlemagne, Paris 2000, benutze ich den Lothschen Text, halte mich aber aus praktischen Gründen an die Ungersche Kapitelzählung.

3 Mlat. Warmatia, häufiger Wormatia.

4 Und damit auch in: La Gran Conquista de Ultramar, Biblioteca Nacional MS 1187, ed. Louis Cooper, Madison 1989.

5 Vgl. die Edition Pascual de Gayangos, Madrid 1858, p. 185 b, nach dem Erstdruck Salamanca 1503. - Francisco Bautista, Sobre la materia carolingia en la Gran conquista de Ultramar y en la Crónica fragmentaria, in: Hispanic Research Journal 9 (2002) 209-226, schlägt für die Aufnahme die Zeit um 1390 vor.

6 Vgl. Gustav Adolf Beckmann, Gualter del Hum - Gaiferos - Waltharius, Berlin 2010, 85 mit n. 109, mit Hinweis auf die Positionen von Menéndez Pidal, Millet und Heintze (dort nachzutra- 
Von dem mittelndl. Gwidekijn van Sassen schließlich ist nur ein Fragment von knapp 200 Versen erhalten. ${ }^{7}$ Es beschreibt einen Ausfall der schon in ihrer Hauptstadt Sassine belagerten Sachsen unter Gwidekijns Bruder, dem Riesen Fledric, der dabei umkommt; es endet mit einer Diskussion der Sachsen darüber, wer die Hiobsbotschaft Gwidekijn überbringen soll, der sich in einer anderen Stadt namens Bacham aufhält. Besamusca betrachtet den Gwidekijn wohl $\mathrm{zu}$ Recht als einen genuin niederld. Text ohne frz. Vorlage. ${ }^{8}$

Somit beschränke ich mich im Folgenden auf die Saisnes und die beiden Texte in der KMS.

\section{b Die Überlieferung der Saisnes}

Um einen wichtigen Schluss nicht zu entwerten, müssen wir auch die Überlieferung der Saisnes skizzieren. Zu Beginn (v. 32 Mss. AR 29 Mss. LT) nennt sich Bodel als Autor; doch Ende 1202 kam er in die Leproserie, und zumindest der Schlussteil des Werkes von reichlich 2000 Versen, der als typischer Fortsetzungsteil von Guitechins Söhnen handelt, fällt literarisch stark ab und kann hier außer Betracht bleiben. Der verbleibende Hauptteil (in AR knapp 4300, in LT etwa 5600 Verse) hat einen in sich befriedigenden Schluss: nach langem, wechselvollem Krieg tötet Karl Guitechin im Zweikampf, zieht in seine Hauptstadt Tremoigne 〈Dortmund ${ }^{9}$ ein, nimmt seine Witwe Sebile ins Christentum auf und verheiratet sie mit seinem Neffen Balduin, der zum Herrn von Sachsen wird; der Deuteragonist Berart erhält Sebiles Vertraute Helissent; Karl zieht mit seinem Heer ab.

Aber auch dieser Hauptteil bietet ein Überlieferungsproblem. Bis v. 3307 AR 3001 LT gehen die beiden Ms.-Familien eng zusammen. Von da bis zum Ausscheiden von A (v. 4337 AR 5713 LT) zeigen sie miteinander keine Wortanklänge mehr und können nicht mehr einem gemeinsamen Autor zugeschrieben werden, da ein solcher doch selbst bei einer Umarbeitung oder Rekonstruktion aus dem Gedächtnis ab und zu «einen Stein auf dem anderen〉 gelassen hätte. Wohl aber zeigen sie inhaltlich noch so viel Parallelismus, dass die Editorin sie weiterhin en regard abdrucken kann. Sie verhalten sich also, als hätten zwei

gen: Ramón Menéndez Pidal, La Chanson des Saisnes, in: Mélanges de linguistique et de littérature romanes offerts à Mario Roques, 4 Bde., Paris 1950, Bd. 1, 229-244).

7 Ediert von Gerrit Kalff, Middelnederlandsche epische fragmenten, Arnheim, 1886, 159-167.

8 Bart Besamusca, Medieval Dutch Charlemagne Romances: An Overview, in: Olifant 26 (2011) 167-193, hier 184.

9 Zum afrz. Namen von Dortmund vgl. unten Abschnitt 7b! 
Autoren nach derselben inhaltlichen Skizze zwei epische Texte verfertigt. Die Editorin sprach 1989 und 1990 Bodel beide Versionen $\mathrm{ab} ;^{10}$ man darf dann wohl schließen, dass der Dichter beim Gang in die Leproserie eine Skizze hinterließ, die zwei Nachfolger 〈um die Wette ausführten. Doch schon 1991 demontierte Povl Skårup ${ }^{11}$ die meisten chorizontischen Argumente der Editorin und tendierte mit aller Vorsicht dazu, die eine Fassung weiterhin Bodel zuzuschreiben; man kann dann etwa annehmen, dass Bodel die Skizze einem Nachfolger hinterließ, aber wider Erwarten in der Leproserie noch den gesamten Hauptteil fertigstellen konnte. Wie dem auch sei, folgt daraus für uns, dass der große Zweikampf zwischen Karl und Guitechin von Bodel zumindest in seiner Skizze geplant war; mit einiger Wahrscheinlichkeit können wir ihn aber auch in seinen Worten lesen.

\section{Der Höhepunkt der Handlung: der kriegsentscheidende Zweikampf Karls und Widukinds}

Dieser Zweikampf sollte uns interessieren. Als Romanist mag man es zunächst für selbstverständlich halten, dass er das Rolandslied nachahmt, hier also das Duell zwischen Karl und Baligant. Doch hat schon Gaston Paris ${ }^{12}$ auf einen viel älteren Zweikampf zwischen Karl und Widukind hingewiesen, freilich ohne ihn in Verbindung mit Bodel zu bringen. Gerade das aber halte ich für lohnend.

Dieser legendäre Zweikampf als Abschluss des Sachsenkrieges findet sich schon in zwei gut datierbaren Texten aus den Jahren 973-980 und 1002-1014: der Vita Machtildis antiquior und ihrer Umarbeitung, der Vita Mathildis posterior, Lebensbeschreibungen der deutschen Königin Mathilde (†968), der Frau Heinrichs I. ${ }^{13}$

Mathildes Heiligkeit beruht hauptsächlich darauf, dass sie das Kanonissenstift Quedlinburg und das Nonnenkloster Nordhausen gründete. ${ }^{14}$ Aus einem

10 Sowohl in ihrer Edition (wie Anm.1) als auch im Begleitband: Annette Brasseur, Étude linguistique et littéraire sur la Chanson des Saisnes de Jehan Bodel, Genève 1990, beide passim. Ähnlich schon dies., La part de Jehan Bodel dans la Chanson des Saisnes, ou quatre rédactions en quête d'auteur, in: Olifant 13 (1988), 83-95.

11 Povl Skårup, Jehan Bodel et les autres auteurs de la Chanson des Saxons, in: Revue Romane 26 (1991) 206-218; vgl. auch dens., L'unité contestée de la Chanson des Saisnes, in: Olifant 20 (1995-1996 [1999]) 7-12.

12 Paris, Gaston, Histoire poétique de Charlemagne, Paris 1865, 292.

13 Editio citanda: Die Lebensbeschreibungen der Königin Mathilde, hg. von Bernd Schütte, Hannover 1994 (MGH SS.schol. 66).

14 Lexikon des Mittelalters (im Folgenden: LM), Studienausgabe, 9 Bde., München 2003, s. v. Mathilde 1. 
von beiden stammt die Vita antiquior, also entweder von einem Priester, der den Kanonissen bzw. Nonnen die Sakramente spendete, oder wahrscheinlicher von einer der geistlichen Damen selbst.

Die Autorin - wenn ich kurzerhand so sagen darf - kennt Terenz' Andria (wie ihre ältere Landsmännin Hrotsvith von Gandersheim), Vergil und geistliche Literatur, von Historikern aber nur die Sachsengeschichte ihres Zeitgenossen Widukind von Corvey. ${ }^{15}$ Insbesondere weiß sie nichts von der Gattung der Annalen, ahnt jedenfalls nicht, wie leicht ihre offensichtlich bona fide formulierten Worte über das Ende von Karls Sachsenkrieg von dort aus hätten widerlegt werden können.

Königin Mathilde war eine Nachkommin des großen Widukind, ${ }^{16}$ für den Karl der Große ja persönlich Taufpate geworden war. Ihre Biographin betrachtete diese Herkunft als hohen Adelstitel; doch um hier jede Zweideutigkeit in Glaubensdingen auszuschließen, musste sie Widukinds Bekehrung [a. 785] erwähnen, die sie bereits mit dem Ende des Sachsenkriegs [a. 804] gleichsetzt. Sie schreibt also gleich in Kap. 1 (p. 113 ed. Schütte):

[Widikindus dux Saxonie], quondam demonum captus errore, praedicatorum pro inopia idola adorans, christianos constanter persequebatur. Illo autem tempore Karolus Magnus arcem tenens imperii, vir christianissimus, armis strenuus, lege eruditus totusque in fide catholicus et erga dei cultores benivolus ac devotus, contra eundem Widikindum bella cum exercitu iniit defendendę causa fidei, ut semper contra paganos solebat.[!] Cumque simul convenissent, utrisque placuit principibus, ut ipsi singuli dimicaturi consurgerent et, cui sors victoriam contulisset, ipsi totus exercitus sine dubio pareret. Quibus congressis ac diu multumque concertantibus [!] tandem dominus, lacrimis pulsatus christianorum, fideli suo bellatori de hoste concessit triumphum, ut fides meruit. Tunc tanta mentis mutatio Widikindi invasit pertinentiam, ut se voluntarius cum familia sua omnique paganorum exercitu tam potestati regis quam fidei submitteret catholicę; quem imperator benigne suscipiens, baptizari fecit a sancto Bonifacio episcopo, ipse eum levans de sacro fonte. ${ }^{17}$

(‘[Herzog Widukind von Sachsen], einst im dämonischen Irrglauben befangen und infolge des Fehlens christlicher Prediger ein Götzenanbeter, verfolgte die Christen ständig. Damals hatte die Kaiserwürde Karl inne, ein zutiefst christlicher Mann, ein starker und aus-

15 Vgl. dazu im Einzelnen Schütte (wie Anm.13) 12, $17 \mathrm{f}$. Die Ed. von Winterfeld (MGH SS.schol. 34, 109-111 und 122) verzeichnet zu Hrotsviths Gallicanus sechs sprachliche Anklänge an die Andria.

16 Diese Aussage der Vita gilt in der modernen Geschichtswissenschaft durchweg als vertrauenswürdig, obwohl die genaue Filiation nicht mehr zu rekonstruieren ist. Selbst wenn sie irrig wäre, würde das die folgende Darlegung nicht tangieren.

17 Schütte (wie Anm. 13) 113. - Die [!] von mir. Zudem habe ich gegen den Editor zur Erleichterung des Verständnisses die Satzzeichen etwas vermehrt sowie $e$ in $e$ verdeutlicht, wo es für ae steht. Die folgende Übersetzung von mir. 


\begin{abstract}
dauernder Kämpfer, in den Gesetzen bewandert, streng rechtgläubig und gegen die Glaubenskünder von frommem Wohlwollen erfüllt. Zur Verteidigung des Glaubens zog er mit seinem Heer gegen Widukind zu Feld, wie er es gegen Heiden immer zu tun pflegte. [!] Als man aufeinandertraf, einigten sich die beiden Fürsten auf einen Zweikampf dergestalt, dass demjenigen, dem das Schicksal den Sieg verleihen werde, alles [scil. das beiderseitige] Heer unbedingt gehorchen solle. Nachdem beide gegeneinander angetreten waren und lange und erbittert gegeneinander gekämpft hatten [!], gewährte endlich der Herr, von den Tränen der Christen gerührt, seinem treuen Kämpen, wie es dessen Glaube verdiente, den Sieg über den Feind. Da ergriff den bisher so starrsinnigen Widukind ein solcher Geisteswandel, dass er sich aus eigenem Antrieb mit seiner ganzen Sippe und dem ganzen Heer der Heiden der Herrschaft des Königs und dem katholischen Glauben unterwarf. Der Kaiser nahm ihn gnadenvoll auf, ließ ihn vom heiligen Bischof Bonifatius taufen und war dabei selbst sein Pate.»)
\end{abstract}

Die Autorin ist sich also schon sicher, dass Karl auch abgesehen vom Sachsenkrieg 〈immer» Kriege für das Christentum zu führen «pflegte». Das hört sich nach mehr an als einer Anspielung auf den einen Avarenkrieg, kann darüber hinaus eigentlich nur den Spanienkrieg meinen; jedenfalls erhält Karls Bild schon epische Züge. Der besiegte Widukind unterwirft sich im letzten Augenblick sfreiwillig, und Karl selbst wird sein Pate bei der Taufe [a. 785], die aber vollzogen sein soll von Bonifacius [Märtyrer a. 754!]. Dass diese bis auf die Patenschaft irrigen Behauptungen, die doch nur aus der Mündlichkeit stammen können, ohne jedes vorsichtige «wie berichtet wird〉 oder ähnlich erscheinen, deutet darauf hin, dass sie schon allgemein geglaubt wurden. In der Folge schildert die Autorin, wie Widukind zum exemplarischen Förderer der Kirche geworden sei; möglicherweise wird dabei vorausgesetzt, dass er unter Karl die Herzogswürde behalten konnte.

Die Vita posterior, erhalten in einem Ms. schon des frühen 11. Jh., ist auf Anregung von Mathildes Urenkel Heinrich II. (1002-1024) entstanden und ihm gewidmet. Dieser, aus der bayrischen Nebenlinie der Ottonen kommend, war nur ein Halbvetter seines Vorgängers Otto III.; die engste gemeinsame Ahnin beider und einzige Heilige in der Familie war Mathilde, so dass das Werk implizit auch der Legitimierung Heinrichs diente. Der Autor hat den vorgefundenen Text in gepflegt-zwangsloseres Latein umgegossen und in unserem Passus nur einige Steigerungen angebracht. ${ }^{18}$ Erstens sollte stärker betont werden, dass der Krieg epische Dimensionen hatte; also zog Karl nicht «mit einem Heere〉, sondern «mit seiner ganzen Heeresmacht gegen Widukind, congregans omnem suum exercitum. Und ihren Zweikampf verabredeten die beiden Herrscher erst, als nach langem Krieg beide Seiten erschöpft waren: Cumque convenissent et

18 Vgl. dazu Schütte (wie Anm. 13) 148 f. 
diu inter se certarent amborum milites et ex utraque parte iam deficerent vires, placuit principibus, ut etc. Zweitens wird eindeutiger gesagt, dass Widukind in Sachsen weiterherrschen durfte. Da der Autor aber die «aus der Vita antiquior bekannte emphatische Verherrlichung der patria Saxonum» merklich zurückdrängt, ${ }^{19}$ stattdessen den bayrischen Zweig der Ottonen herausstellt, saß er dem Editor zufolge nicht in Sachsen, sondern gehörte zu Heinrichs Umgebung; dazu passt übrigens die hochdt. Lautform Witi- statt des niederdt. Widu-der Volage. Wenn die Vita posterior somit von einem Herrscher bestellt war, dessen Hausmacht in Bayern lag und der zudem geistliche Bildung genossen hatte wie Heinrich, und wenn sie trotzdem die ältere Erzählung nicht nur beibehalten, sondern noch steigern konnte, zeigt sie noch klarer als diese, wie sehr im breiten Publikum, offenbar weit über Sachsen hinaus, an ein für Karl siegreiches Duell mit Widukind als Ende des langen, kräfteverschleißenden Krieges geglaubt wurde.

Solch ein für Karl siegreiches Duell als Kriegsende war auch für französisches Empfinden akzeptabel - siehe Bodel. Wenn wir uns angesichts dessen von der apriorischen Meinung lösen, dass epische Motive nicht über Sprachgrenzen wandern, ist nicht einmal auszuschließen, dass der Duell-Gedanke in der Frankophonie entstanden ist und in Sachsen nur den Schlenker von Widukinds Weiterherrschen bekommen hat. Jedenfalls darf man bezweifeln, dass die Annahme, das Duell-Motiv sei zweimal entstanden - was die heutige Forschung voraussetzt, ohne sich dessen bewussst zu sein - mit dem Prinzip der Denkökonomie vereinbar ist. Was die Zeitspanne angeht, die zwischen den Viten und Bodel liegt, darf man nicht vergessen, dass Bodel ein älteres Epos erneuerte. Denn er sagt einleitend in v. 27-32 AR 24-29 L(T), dass nicht nur cil bastart jougleour von Guitechin schon si com par asseniaus singen, also sau hasard, à l'aveuglette,${ }^{20}$ sondern auch, dass selbst, wer es genauer weiß, besser schweigt, weil er nicht die neuen «reichen〉 Verse zu machen versteht wie eben Jehan Bodel; der Stoff lag also zumindest in den Grundlinien und in weniger «reichen〉 Versen Bodel schon vor.

Worin sich allerdings Frankreich und Deutschland unterschieden, ist Widukinds Schicksal: in allen drei Fassungen der frz. Sachsenepik kommt er zu Tode. Ich halte auch das für einen indirekten Reflex der Geschichte, hier der Mentalitätsgeschichte. Das 10. und das 11. Jh. waren für Frankreich eine Zeit der schweren Demütigung durch Deutschland: ein Heinrich I. aus jenem Sachsenstamm, den niedergezwungen zu haben einen Großteil von Karls des Großen Ruhm ausmachte, entriss den Franzosen die ganze Lotharingia von der Nordsee bis in die

19 So Schütte (wie Anm. 13) 44.

20 So die Editorin im Glossar, dazu ihre Erklärung in der note zu v. 29/26. 
Vogesen mit ihren beträchtlichen frankophonen Teilen; beginnend mit seinem Sohn Otto, der zudem Karls Kaiserkrone usurpierte, waren sogar leibliche Nachkommen Widukinds gleichzeitig arbiter und Nutznießer der französischen Selbstzerfleischung zwischen Karolingern und Kapetingern, wobei im Bedarfsfall wie 978 ein sächsisch dominiertes, aus romanischer Sicht also halbbarbarisches Heer vor Paris erscheinen konnte; ihre salischen Nachfolger schließlich annektierten 1033 auch das rein gallorom. Burgund von Besançon bis Arles. Da die Kaiserkrone bei Deutschland verblieb, ebbte der französische Albtraum selbst im 12. Jh. nur langsam ab, um erst nach Bodels Zeit mit Bouvines (1214) und Tagliacozzo (1268) ganz zu verschwinden. Für die Jahrhunderte der Demütigung rächte sich die Kollektivphantasie: einen Widukind, der die Wurzel all dieses Übels war, durfte Karl nicht am Leben lassen.

Man könnte einwenden wollen, die beiden Erzählungen in der KMS enthielten das Duell nicht; das mindere die Chancen der Kontinuität zu Bodel. Doch betrachten wir in beiden den jeweiligen Schluss genauer!

Die KMS I gibt, wie bei den meisten in ihr behandelten Epenstoffen, auch in Kap. 46-47 nur den gedrängten inhaltlichen Abriss (etwa zwei heutige Druckseiten!) eines afrz. Epos. Hier der Schluss: nur Tremoigne 〈Dortmund〉 ist noch zu erobern. Als Roland das Horn zum Sturmangriff bläst, erneuert Gott sein Jericho-Wunder. En KarlaMagnus for $j$ borgina ok allt lid hans, ok drapu Vitakind kong [...] «Und Karl der Große drang in die Stadt ein und all sein Volk, und sie töteten König Vitakind.> Das Jericho-Wunder ist zwangsläufig eine sekundäre - fromme, aber auch fazile - Zutat zu einer präexistenten Erzählung, ganz so, wie es das oft schon bei Historikern der Völkerwanderungs- und Merowingerzeit, dann in der afrz. Epik z. B. beim Fall sowohl von Lucerna Ventosa als auch von Nobles/Grenoble im PT oder bei der Einnahme von Narbonne im Roland-V4 ist. ${ }^{21}$ Es bringt aus der Bibel die Vorstellung mit, dass Gott die Verteidiger der Stadt zur Niedermetzelung freigibt: ein ganzes Heer dringt gleichzeitig ein, «ein Schlachten war's, nicht eine Schlacht, zu nennen.» Eben dadurch wird ein faires, leidlich ausgedehntes Duell zwischen zwei großen Kämpfern ohne Intervention Dritter, so es denn vorher der Erzählung angehörte, narrativ unmöglich. Es ist zwar immer noch wahrscheinlich, dass der Erzähler des vollen Epos wenigstens den Todeshieb auf Widukind nicht gegen alle epische Technik einem anonymen Kollektiv überließ, sondern für Karl reservierte, doch in der Zusammenfassung kann daraus das oben zitierte Textstück geworden sein. Als in Kap. 55 ein kurzer Rückbezug auf Kap. 46f. nötig wird, heißt es denn auch einfach im Singular: ‘als König Karl die Stadt Dortmund eingenommen und den König Vitakind getötet hatte> - ohne Erwähnung eines Heeres.

21 Vgl. im Einzelnen Rajna, Pio, Le origini dell'epopea francese, Firenze 1884, 247-249. 
Anders als die KMS I will die KMS V zwar einen afrz. Text prinzipiell im Verhältnis 1:1 wiedergeben. Doch erlaubt sie sich zumindest einen schweren Erzählbruch, welcher exemplarisch zeigt, wie ungeniert und zugleich desaströs deren Redaktor mit seiner Materie umsprang. Obwohl in Kap. 40 Guiteclins Frau Sebile bereits Gebete an den Christengott richtet und obwohl in Kap. 44 sie und Balduin einander mehr oder minder ihre Liebe gestehen, empfindet sie im Schlusskapitel 55 über die Gefangennahme Guiteclins tiefsten Schmerz und entflieht mit ihren beiden Söhnen, und zwar, ohne dass man von allen dreien je Weiteres erfährt. ${ }^{22}$ Bei einem solchen Redaktor muss man auch mit anderen Eingriffen rechnen, so auch bei dem uns interessierenden Kapitel 54. Die Entscheidung des Krieges bringt auch hier ein Zweikampf Guiteclins, aber mit Balduin. Schon haben die beiden einander aus den Sätteln geworfen, da ermahnt Balduin Guiteclin, sich zu ergeben, und - Guiteclin ergibt sich; doch ohne Gnade zu finden: er wird (Kap. 55) nach Paris gebracht und dort zu lebenslänglichem Kerker verurteilt. Ein Duell mit Widukind als essenzieller Abschluss des Krieges ist also da wie in den Mathildenviten und in den Saisnes. Da muss man damit rechnen, dass in der KMS V Balduin als der im Aufstieg begriffene Protagonist der Sachsenepik an die Stelle Karls getreten ist. ${ }^{23}$

22 Angesichts eines solchen Befundes versteht man den Grundtenor der Arbeiten von Hélène Tétrel, Le brouillage des sources dans les adaptations norroises des chansons de geste, in: Le Vrai et le Faux au Moyen Âge. Textes réunis par Élisabeth Gaucher, Villeneuve d'Ascq, 2005, 195-207, und La Chanson des Saxons et sa réception norroise. Avatars de la matière épique, Paris 2006: sie glaubt bei der KMS V und tendenziell überhaupt bei der KMS nicht an einheitliche afrz. Vorlagen und damit deren altnord. (Quasi-) «Übersetzungen`, sondern an einen «brouillage des sources» und möchte die Epik prinzipiell, auch stofflich, als eine «mouvance», eine in ständiger Durchknetung befindliche Erzählmaterie, ansehen. Ich kann hier diese These nicht im Einzelnen untersuchen; immerhin sei festgestellt, dass Tétrel für die KMS V (und wohl auch für X) gute Argumente hat, Recht auch darin, dass es nicht einfach zwei Sachsenepen das von Bodel und ein zweites - gegeben hat und dass nicht die außer-Bodelsche Sachsenepik genetisch von vornherein älter als Bodel ist; doch bedürfte ihre These für die anderen Branchen der KMS genauer Nachprüfung, wobei die Beweispflicht grundsätzlich bei den Chorizonten und nicht bei den Unitariern läge. Für die KMS I ist vielmehr an einer einheitlichen frz. Vorlage festzuhalten (die allerdings ihrerseits Epeninhalte aufreihte, dazu Gustav Adolf Beckmann, Die Karlamagnús-Saga I und ihre altfranzösische Vorlage, Tübingen 2008, passim), ebenso für die KMS VIII bis zum Beginn der (dort fehlenden) Baligant-Schlacht (dazu Paul Aebischer, Rolandiana Borealia, Lausanne 1954, und Eyvind Fjeld, Halvorsen, The Norse Version of the Chanson de Roland, København 1959, beide passim).

23 Balduin, Protagonist in KMS V und Saisnes, ist in dieser Funktion fast mit Sicherheit schon dem Rolandslied (v. 313) bekannt; denn dort ist Ganelon überzeugt, dass er ein prozdom wird, was die frz. Epik nur im Sachsenkrieg thematisiert. Auch der PT (Kap. 11) nennt ihn in der Liste von Karls pugnatores maiores zwischen Salomon, dem Freund des Estout von Langres, und dem Friesen Gandelbod, assoziiert ihn also wohl mit dem Nordosten des Karlsreiches, was zum Protagonisten eines Sachsenkrieges passt. Weshalb er in der KMS I fehlt, ist unklar. 
Die beiden nordischen Fassungen sprechen also nicht dagegen, dass zwischen den Mathildenviten und Bodel eine Kontinuität des Duells zwischen Karl und Widukind als des kriegsentscheidenden Schlusses besteht; vielmehr erweist sich damit der zu Bodel führende Überlieferungsstrang als der konservativere und in gewissem Sinn - da Bodels Dichtung erhalten geblieben ist, die Vorlagen von KMS I und V aber nicht - als der Hauptstrang. Doch dieses Duell ist nur der Schlussakkord einer komplexen Handlung, deren Konstante ein Fluss ist. Ihr wenden wir uns jetzt zu.

\section{Der Fluss als Strukturachse der Handlung: Grundlagen und Anfänge}

Die Bedeutung der Flüsse für die Sachsenepik hat im Prinzip schon Rajna erkannt. ${ }^{24}$ Doch da man diese Bedeutung heute präziser herausarbeiten kann, sei das hier versucht.

Durch Stammes-Sachsen, also grosso modo Nordwestdeutschland, ziehen sich relativ viele, meist aus den regenreichen Mittelgebirgen kommende Flüsse, und zwar außer Ruhr und Lippe jeweils zur Nordsee hin. Ein aus dem Westen oder Südwesten kommender Invasor musste sie einen nach dem anderen überwinden, was im Mittelalter keine kleine Aufgabe war. Selbst in der Galloromania gab es zwischen der Spätantike und dem Jahr 800 so gut wie keinen Brückenbau; ${ }^{25}$ man behalf sich mit der römischen Substanz und wohl einem Minimum an Reparaturen. In Sachsen gab es keine römische Substanz, somit außer gewiss Holzstegen über kleinere Gewässer - keine Brücken. Durch manche Flüsse gab es Furten; weil sie aber von einem Heer nur in schmaler Linie durchquert werden konnten, bildeten selbst sie eine Gefahr: wegen mangelnder Ausweich- und Deckungsmöglichkeiten war das Heer ein leichtes Ziel für einen Pfeilregen; brachte dieser einige Pferde oder Menschen zu Fall, so war die Furt verstopft und alles Weitere Zufall. Wo über größere Flüsse Fähren den Verkehr, insbesondere den Handel, aufrecht erhielten, ließen sich diese beim Herannahen eines Angreifers in kürzester Zeit verbergen oder zerstören. Pferde schließlich können zwar schwimmen, verweigern es aber oft, dabei eine Last oder einen Menschen, erst recht einen gepanzerten Reiter, zu tragen. So konnten die Flüsse in Sachsen fremde Heere zumindest merklich aufhalten und wurden

24 Rajna (wie Anm. 21) 266-268.

25 Erst nach 800 setzte Brückenbau in allmählich zunehmendem Maße ein. Ich habe an anderer Stelle einen detaillierten Überblick über Brückenbauten in Deutschland und Frankreich bis 1230 gegeben; vgl. Beckmann (wie Anm. 22) 166 Anm. 226. 
damit zum strategischen Problem für jeden Invasor und zur natürlichen Verteidigungslinie für jeden Verteidiger.

In den Sachsenkriegen des historischen Karl spiegelt sich das so, dass die Franken es teils dabei bewenden ließen, einen Fluss erreicht zu haben, teils an Flussübergängen in heftige Kämpfe gerieten: 772 gelangte Karl bis zur Weser, 775 erzwang er kämpfend erst den Ruhr-, dann den Weserübergang (multi Saxones ibi occisi sunt, sagen die Reichsannalen) und gelangte bis an die Oker; im gleichen Jahr gelang den Sachsen kurzzeitig bei Lübbecke ein Weserübergang westwärts, und Karl selbst musste herbeieilen, um sie an die Weser zurückzutreiben (vgl. zu dieser Episode auch die aufrichtigere bis 829 reichende Fassung der Reichsannalen!); 776 gelangten die aus der Hohensyburg ausbrechenden fränkischen Truppen bis zur Lippe; 782 zog Karl bis zur Aller nach Verden und hielt dort sein Blutgericht; 795 gelangte er erstmalig bis zur Elbe; 797 zog er nur bis zur Weser bei Herstelle, aber um dort demonstrativ zu überwintern, im folgenden Frühjahr weiter bis zur Elbe. Schließlich zog er, nunmehr bei drohenden Dänenkriegen, 804 bis zur Elbe und 810 nochmals bis zur Weser an der Allermündung (vgl. jeweils die Reichsannalen).

Solche Vorgaben der Realität machten nun jeweils einen Fluss auch zum klassischen, fast obsessionshaften Schauplatz der Sachsenepik, zum roten Faden, wie er sich durch alle drei zu betrachtenden Texte zieht - und in dieser literarischen Funktion schon viel älter als die drei Texte ist.

Denn hierher gehört schon die Story, die der um 727 geschriebene Liber Historiae Francorum von einem (mit einiger Wahrscheinlichkeit legendären) Krieg des Frankenkönigs Chlothar II. und seines Sohnes Dagobert I., damals erst Unterkönigs in Austrasien, irgendwann zwischen 623 und 629 gegen die Sachsen erzählt: ${ }^{26}$ Dagobert gelangt bis an die Weser, wird aber am Kopf verwundet und ruft durch Übersendung einer blutigen Locke seinen Vater mit Verstärkungen herbei; aber auch jetzt setzt Chlothar erst über den Fluss, nachdem ihn der Sachsenführer durch den Schimpfruf bale (var. blare) iumente aufs Äußerste beleidigt hatte. ${ }^{27}$ Das Kapitel des Liber mit dieser Erzählung wurde

26 Liber Historiae Francorum, cap. 41 (MGH SS.mer. 2.404 f.). Die Vermutung der Legendarität stützt sich nur darauf, dass der ältere, nämlich schon um 660 schreibende (Pseudo-) Fredegar von dem Krieg nichts weiß; sie ist trotzdem in der Romanistik seit Bédier allgemein rezipiert. Die Historiker sind sich nicht ganz so einig: beispielsweise hält Bernard S. Bachrach, Merovingian Military Organization 481-751, Minneapolis, Univ. of Minnesota Press, 1972, 85 f., sie kommentarlos für wahr.

27 Diese zweigliedrige Beleidigung ist meines Wissens nirgends voll befriedigend erklärt (z. B. entgeht jeweils ein wichtiger Teil der Pointe sowohl Gerhard Eis, Kleine Schriften zur altdeutschen weltlichen Dichtung, Amsterdam 1979, 10-13, als auch Wolfgang Haubrichs, Geschichte der deutschen Literatur , I/1: Die Anfänge, Tübingen 1995, 100). Hier in gedrängter Form meine Interpretation: Da im Merowingerreich nur die Angehörigen der herrschenden Dynastie, als 
von Gaston Paris nur nebenbei aus einem anderen Grunde erwähnt; doch Rajna behandelt es sehr eingehend und hält es für ein sicheres Zeugnis merowingerzeitlicher epischer Schöpfung, lässt aber gerade wegen der Ähnlichkeit der realen Situationen die sich anschließende Frage, ob eine Kontinuität zur afrz., (stofflich) karolingischen Sachsenepik besteht, nahezu offen (einer positiven Antwort kommt er p. 268 n. 5 nahe). ${ }^{28}$ Bédier leugnet bekanntlich nicht nur eine

sog. reges criniti, langes Haar tragen durften, ist die blutige Locke für Chlothar kein dunkles Symbol, sondern ein nüchtern-eindeutiges Beweisstück: sie kann nur von einer Kopfverwundung seines Sohnes stammen. Bei den damaligen Rezipienten der Story ist damit für das Folgende die optische Vorstellung des rex crinitus präsent gemacht. In der Beleidigung nun ist iumente grammatisch falscher, aber psychologisch einleuchtender Vokativ zu lat. iumentum (< iug-mentum) 〈Lasttier (Pferd, Esel oder Maulesel)〉; weil gegenüber dem Streitross weitaus geringer geschätzt, wäre schon dies, auf einen Menschen angewandt, eo ipso beleidigend. Nach dem FEW (s.v.) hat jedoch «im Gallorom. [...] diese Bedeutung nur in Randgebieten weitergelebt», während das Wort bereits in der Lex Salica [hier allerdings noch umstritten], den Leges Alamannorum [frühes 8. Jh., hier eindeutig; so auch das MLLM s. v.] und in einem Kapitular Karls d. Gr. «Stute〉 bedeutet; vgl. ferner TLL 7.2 col. 647.44-52, wo diese Bedeutung auch in den Leges Visigothorum [in diesem Teil 7./8. Jh.] nachgewiesen wird. Sie liegt offenbar auch an unserer Stelle vor; denn erst dadurch wird die Beschimpfung treffend: als langhaariger rex crinitus unter lauter Männern mit gekapptem Haar wirkt der Merowinger auf den Sachsenführer erstens weibisch; doch zweitens erinnert ihn sein sorgfältig in der Mitte gescheiteltes Haar (wie dies bei den Merowingern laut Agathias hist. 1.3.4 üblich war) an eine Pferdemähne; ergo $\langle$ weiblich $\rangle+\langle$ Pferd $\rangle=\langle$ Stute $\rangle$ - Von bale behauptet DuCange s. v. balejumentum ohne Quellenangabe, es sei vetus Gallicum und bedeute sfalsch;; aber ein solches Wort ist im Afrz. nicht zu finden, und Holder, Alt-celtischer Sprachschatz, bietet nichts für das Gallische (oder eine andere kelt. Sprache). Das Mlat.Wb. s.v. balus (2) kennt nur unseren Beleg, schlägt als Bedeutung «?falsus〉 vor und verweist auf ahd. balo. Doch nach dem Ahd. Wörterbuch von Frings/ Karg-Gasterstädt ist balo immer Subst.: 〈das Übel, das man erleidet oder tut〉. Zudem: wäre die Bedeutung des Wortes bale hier einfach «übel, bösartig», so sollte man Übersetzung ins Lat. erwarten; denn auch iumente ist ja im Munde des Sachsen schwer vorstellbar, ist also Übersetzung ins Lat.(-Rom.). Es muss somit ein stärker situationsspezifisches, weniger leicht ins Lat. übersetzbares Wort vorliegen, und da Chlothar durch Abnehmen des Helms soeben seine crines cum canicie variatas, seine langes, aber grau- bis weißmeliertes Kopfhaar, gezeigt hat, ist eine entsprechende Farbbezeichnung zu erwarten, insbesondere eine auf Pferde angewandte. In der Tat weist das FEW, Bd. 1, s. v. balla (germ.) (weißgefleckt), hin auf eine Wortsippe got. bala, engl. ball «Pferd mit einer Blässe` [= Blesse, weißem Stirn- oder Nasenfleck], kymr. bal having a white face or forehead (as a horse), ähnlich bret. bal; ob sie letztlich germ. (so von Wartburg) oder kelt. ist (so andere), kann uns gleichgültig sein. Auch dass sie in afrz. baille 〈cheval marqué d'une tache blanche au dos〉 (> mbret. baill) nur noch mit einer $j$-Erweiterung vorliegt, ist kein Gegenargument. Liegt ein solches Wort vor, so wird auch verständlich, dass die Mss. A3a ${ }^{1}$ (9. Jh.) und B2 $\mathrm{c}^{1}$ (10. Jh.) es unabhängig voneinander durch blare ersetzen; denn dies gehört klar zu afrz. bler «(Pferd) nicht genau bestimmbarer Farbe, (Tier) mit weißer Stelle am Kopf;; vgl. FEW, Bd. 1, s. v. *blaros.

28 Paris (wie Anm. 12) 443; Rajna (wie Anm. 21) 111-130, 261-268. 
merowingisch-karolingische epische Kontinuität, sondern auch die Existenz einer romanophonen merowingerzeitlichen Epik selbst, kommt dabei aber auf die Erzählung nur sozusagen als Hintergrund zum Farolied in seiner diesem gewidmeten langen Diskussion. ${ }^{29}$ Ich selbst halte hier wie Rajna Kontinuität für unbeweisbar, aber auch bei weitem nicht für ausgeschlossen, da jeder neue fränkische Flussübergang in Sachsen das Interesse an der Story auffrischen musste. Für Kontinuität könnte insbesondere die Tatsache sprechen, dass auch später zur Sachsenepik das Rufen über den Fluss gehört (KMS V 44, Saisnes v. 1566-1601 und 2217-2291 AR 1496-1524 und 1973-2044 LT), ebenso, wie schon Rajna sah, das episodenhaft-tollkühne Überqueren des Flusses durch Einzelne oder kleine Gruppen. Liegt keine Kontinuität vor, so ist umso mehr $\mathrm{zu}$ bestehen auf der Rolle der Naturgeographie und der mit ihr verbundenen, sozusagen summativen negativen Erfahrung der Franken mit den Flüssen zwischen ihnen und den Sachsen. Auch dann schöpft die Epik von Karls Sachsenkrieg also aus der Realität, sie ist «welthaltig〉; ihre Fluss-Faszination ist nicht Produkt einer realitätsfern arbeitenden je individuellen Phantasie.

\section{Der Beginn der Handlung in KMS I, KMS V und den Saisnes}

In allen drei Sachsenkriegs-Darstellungen beginnt die Handlung mit einem Überfall Widukinds - in der KMS I auf die (anachronistisch) schon christliche Stadt Münster, in der KMS V und den Saisnes auf Köln; Karls Vergeltung bis zur Vernichtung Widukinds ist dann das eigentliche Thema der Erzählung.

In der KMS I hat Karl Roland und Olivier gerade zur Belagerung von Nobles nach Spanien geschickt; da erreicht ihn noch in Aachen die Nachricht von dem sächsischen Überfall. Von dort bricht er mit seinem Heer, zunächst ohne Roland und Olivier, zum Sachsenkrieg auf. In der KMS V ereignet sich der sächsische Überfall in der Frühphase von Karls Spanienkrieg; Karl kehrt von den Pyrenäen aus eilig um, nur Roland bleibt eigensinnig bei der Belagerung von Nobles zurück. In den Saisnes schließlich kehrt Karl mit dem erschöpften Heer soeben aus Spanien zurück; erst die Nachricht von Rolands und Oliviers Tod gibt Guitechin den Mut zum Überfall. Sowohl in der KMS V wie in den Saisnes haben die Sachsen vor Karls Ankunft Köln wieder geräumt.

In der realen Geschichte drangen zu Karls Zeit nur damals, also 778, die aufständischen Sachsen je bis zum Rhein vor, und zwar unter Führung Widukinds bis (Köln-) Deutz, konnten allerdings den Fluss nicht überqueren; ${ }^{30}$ nach

29 Joseph Bédier, Les légendes épiques, 4 Bde., ²Paris, 1926-1929, 4.289-335.

30 Nach den Reichsannalen war Widukind a. 777 mit wenigen anderen zu den «Nordmannen` (hier: Dänen) geflohen, veranlasste aber im folgenden Jahr den Aufstand (per suasionem supra- 
dem Chronicon Moissiacense (bis 818) erreichte die Nachricht vom Sachsenaufstand Karl adhuc in Hispania degentem, dürfte also Karls schwachen Kompromiss mit Saragossa und seinen folgenden Rückmarsch nach Frankreich mit ausgelöst haben. ${ }^{31}$

Alle drei epischen Darstellungen lehnen sich hier also an die Historie an, am relativ engsten die KMS V, am lockersten Bodel. Indem dieser die gesamte Handlung erst nach Roncevaux beginnen lässt, können bei ihm die neuen Helden Balduin und Berart - anders als in KMS I und V - ohne Konkurrenz durch Roland und Olivier die Sympathie des Publikums ganz auf sich konzentrieren, was der Einheit des Werkes zugute kommt. Bemerkenswerterweise wird die essenzielle Gleichzeitigkeit von Karls Sachsenkrieg und seinem Spanien-Abenteuer auch im Rolandslied erinnert: Roland hat für Karl auch Sachsen unterworfen (v. 2330), aber die Sachsen werden sich als erste gegen Karl empören (v. 2921), und zwar - wie bei Bodel - als Folge von Rolands Tod; erst in dem dann folgenden Krieg kann sich auch Balduin als der prozdoem erweisen, als den Ganelon ihn im Geiste schon sieht (v. 314).

Insgesamt hält damit die afrz. Epik etwas historisch durchaus Relevantes fest: es war tatsächlich die Gleichzeitigkeit seiner beiden großen Ambitionen, der Christianisierung Sachsens und der Re-Christianisierung Spaniens, die Karl vorübergehend in Schwierigkeiten brachte.

\section{Der Fluss als Strukturachse der Handlung, insbesondere der Bau einer Brücke}

In den beiden Darstellungen der KMS wird nun schon der Rhein zur Strukturachse; denn gleich jenseits davon beginnt das Feindesland. Und Karl hat die

\footnotetext{
dicti Widochindi) und blieb dessen Seele bis zu seiner Kapitulation a. 784. - Ähnlich wie 778 hatten die Sachsen 556 zu Chlothars I. Zeit kurz den Rhein bei Deutz erreicht, aber nicht überqueren können (Gregor von Tours 4.16, Fredegar 3.52).

31 So speziell Ramón Menéndez Pidal, La Chanson de Roland et la tradition épique des Francs, 2Paris 1960, 201 mit Anm. 1. Dass das Chronicon Moissiacense höchstwahrscheinlich im Recht ist, sieht man an einem Umstand, der sogar Menéndez Pidal entgangen ist. Laut der Vita Hludovici des Anonymus (Kap. 2) hatte Karl auf dem Hinweg nach Spanien essenziell die spätere Pilgerstraße Nr. 1 Tours-Ostabat benutzt und seine hochschwangere Frau Hildegard bis in die Pfalz Chasseneuil (dicht südlich Poitiers) mitgenommen (von Herstal $700 \mathrm{~km}$ !); das hat Sinn nur, wenn er plante, sie auf dem Heimweg, nunmehr als stolzer Vater, wieder dort abzuholen. Dazu hätte er bei seiner Rückkehr auch alle Ursache gehabt; denn Hildegard hatte inzwischen männliche Zwillinge zur Welt gebracht. Aber der erste Ort in Frankreich, wo wir Karl finden, ist Auxerre (Reichsannalen zu a. 778), nur etwa $50 \mathrm{~km}$ von Vézelay entfernt, dem End- bzw. Ausgangspunkt der späteren Pilgerstraße Nr. 2 Vézelay-Ostabat; Karl hat somit im
} 
größte Mühe, ihn mit seinen Truppen zu überqueren: er muss eine Brücke bauen.

\section{a In der KMS I}

In der KMS I 46 holt er sich dazu Helfer aus seinem ganzen Reich: die Lombarden und die Pizaramenn (am ehesten 〈Pisaner», also Toskaner) ${ }^{32}$ behauen Steine und bringen sie an ihre Stelle, die Burgunder fabrizieren Werkzeuge, ${ }^{33}$ die Bayern liefern Eichenholz, ${ }^{34}$ die Ardenner 〈bauen〉 die Brücke (also wohl ihre Holzteile), alle anderen «helfen〉.

Auch das ist der Geschichte abgeschaut. Da es seit der Spätantike keine Rheinbrücke mehr gab, baute Karl eine bei Mainz, und für Einhart (Vita Karoli 17) war sie nach der Kirche in Aachen seine zweite bewundernswerte Bauleistung, per decem annos ingenti labore et mirabili opere errichtet. Doch da zumindest die normalerweise oberhalb der Wasserlinie liegenden Teile der Brücke aus Holz gefertigt waren, brannte sie ein Jahr vor seinem Tode ab; Einhart zufolge plante er sofort, sie als rein steinerne Brücke wiederzuerrichten, kam aber nicht mehr dazu. Dieser Brückenbau prägte sich den Zeitgenossen zutiefst ein; denn zwei Generationen nach Einhart erfahren wir von Notker Balbulus, der ja aus mündlicher Überlieferung schöpft, dass den Bau tota Europa communi quidem, sed ordinatissimae participationis opere perfecit ‘ganz Europa in gemeinsamer, aber wohlgeordneter Arbeit vollendete> (Gesta Karoli 1.30, Übs. Reinhold Rau). Das kann nur heißen: Karl zog mehrere, auch weit auseinanderliegende Reichsteile zur Arbeit heran; und so, wie er für seine Kirche in Aachen nicht nur die architektonischen Ideen, sondern sogar den Marmor aus Italien, dem Land der erhaltenen antiken und der weiterentwickelten christlichen Steinbauten, bezog, so muss er sich auch für die steinerne Substruktion der Brücke als Steinbau-

Wesentlichen diese benutzt, konnte sich also den Umweg über Chasseneuil nicht leisten. Dies muss er spätestens in Ostabat gewusst haben, und damit höchstwahrscheinlich auch schon, als er zwei Tagesmärsche früher Spanien verließ. Verlängert man die gerade Linie OstabatAuxerre, so zeigt sie nicht auf Karls Ausgangspunkt Herstal, sondern auf den Rhein bei Köln; kurzum: Karl eilte von Ostabat den Sachsen entgegen.

32 Vgl. Beckmann (wie Anm. 22), 167.

33 Die Produktion von Eisen und Eisenwaren war zwar weit stärker dezentralisiert als heute; doch gibt es Anzeichen für frühe Aktivitäten dieser Art sowohl in Nordburgund als auch in Südburgund um Vienne (vgl. z.B. Gustav Adolf Beckmann und Erika Timm, Wieland der Schmied in neuer Perspektive, Frankfurt am Main 2004, 24 mit Anm. 30).

34 In den Alpen findet sich natürlicher Eichenbestand bis in eine Höhe von 1000 m, in den Südalpen bis $1100 \mathrm{~m}$. 
Spezialisten (Nord-) Italiener geholt haben. Sagt die KMS I etwas Anderes? Weil nun Notkers Gesta Karoli immer nur in derselben Verstümmlung (wahrscheinlich ohne den Anfang, sicher ohne den Schluss) erhalten sind, weil ferner ihre handschriftliche Überlieferung - im krassen Gegensatz etwa zu Einharts Vita Karoli - erst im frühen 12. Jh. einsetzt und, soweit sich der Ursprung der Handschriften feststellen lässt, mit Ausnahme eines Ms. aus Clairvaux den alem.bair. Raum nicht überschreitet, ist es relativ unwahrscheinlich, dass das Motiv in die KMS aus den Gesta gelangt ist; eher scheint ununterbrochene mündliche Überlieferung vorzuliegen. Damit stellt sich die Frage, wann es in den Sachsenkrieg-Kontext eingebracht wurde, dem es ja ursprünglich nicht angehörte. Man darf vermuten, dass das sehr früh geschah; denn erstens pflegt sich ceteris paribus ein isoliertes Motiv weniger gut zu halten als ein narrativ verankertes, zweitens wird uns das Motiv auch in KMS V und Saisnes begegnen, nicht nur strukturtragend als «Karls Brückenbau〉, sondern auch mit Erwähnung der vielen Völkerschaften.

In der KMS I bauen diese Völker drei Jahre an der Brücke, dann kommen Roland und Olivier dazu und bringen als gute Organisatoren den Brückenbau in weiteren sechs Monaten zum Abschluss; der eigentliche Feldzug kann beginnen.

\section{b In der KMS V}

In der KMS V mit ihren 55 Kapiteln zieht sich der Bau der Brücke hin von Kap. 16 bis Kap. 36. In Kap. 21 sehen wir auch hier die verschiedenen Völkerschaften bei der Arbeit. Die Römer (in Fassung B,b verflacht zu «seine Armee») erhalten wieder eine Aufgabe für Spezialisten: sie sollen die genaue Breite des Flusses und seine jeweilige Tiefe ausmessen; aber die Sachsen bringen ihnen solche Verluste bei, dass die Überlebenden die Flucht ergreifen. Nun sollen, neben 〈anderen〉, die Alimans (‘Alemannen〉 oder schon «Süd- und Mitteldeutsche`?) weiterbauen, doch nach abermaligen Verlusten beschließen sie zu desertieren; ein nachgeschickter Ritter holt sie unter Drohungen zurück. Als auch die Flamen scheitern, will Karl den Bau schon aufgeben. Da kommen (Kap. 22) zwei Christen aus Spanien und veranlassen ihn, ein Schiff mit Türmen für Armbrustschützen zu bauen und eine hohle eherne Statue daraufzustellen, die, mit Karls Kleidern angetan, ihm täuschend ähnlich sieht und in der ein Mann sitzt, der den Arm der Statue samt Karls Szepter bewegen kann; der drohende eherne Karl erweist sich als immun gegen den sächsischen Pfeilregen, und die Sachsen, entsetzt über das vermeintliche Wunder, ergreifen die Flucht. Man sieht, hier huldigt der Autor jener literarischen Vorliebe für fiktive hochtechnische Kunstwerke, die in der afrz. Literatur mit den Antikenromanen (Eneas, Troie) 
einsetzt; und da man nach mittelalterlicher Überzeugung dergleichen (Quasi-) Zauberwerk in Toledo lernt, kommen dessen Adepten auch in der KMS V aus Spanien. Nun könnte der Brückenbau weitergehen; doch (Kap. 27) Guiteclin selbst, aus anderem Holz geschnitzt als die Flüchtigen, setzt durch, dass an der Stelle, wo sich das Ende der Brücke abzuzeichnen beginnt, schnell ein sächsisches Fort entsteht! Seine Besatzung vernichtet nicht nur das große Schiff, sondern bringt den Brückenbauern erneut so starke Verluste bei, dass Karl wiederum auf den Weiterbau verzichtet. Jetzt (Kap. 29) durchreiten Roland und sein gerade eingetroffener Bruder Balduin mit achthundert Rittern den Rhein und greifen die Besatzung des Forts von hinten an; diese flieht. In Kap. 36 ist die Brücke endlich fertig; doch es nimmt eine ganze Woche in Anspruch, auf ihr Karls Heer, nach Völkerschaften geordnet, über den großen Fluss zu bringen.

Dieser ist Strukturachse der Handlung aber nicht nur durch das, wie man sieht, ergiebige Motiv der Brücke, sondern komplementär dazu auch durch die narrativ nicht weniger ergiebigen Wagnisse derer, die ihn inzwischen zu Abenteuern auf der feindlichen Seite hin und zurück überqueren.

Hierher gehört eine lange Unternehmung Balduins und Berarts (Kap. 2935), bei der sich auch die Liebesgeschichte zwischen Balduin und Guiteclins Frau Sebile anzuspinnen beginnt. Schon vorher (Kap. 17-19) hat Roland eine Überquerung gewagt: ein Eremit offenbarte nämlich dem Erzbischof Turpin eine Furt durch den Rhein, die er erst am selben Morgen entdeckt hatte, als Hirsche dort den Fluss durchquerten; Roland setzt sofort mit einer kleinen Truppe über, wird aber verwundet und hat angesichts einer feindlichen Übermacht alle Mühe zurückzugelangen. ${ }^{35}$ Damit diese Furt die Notwendigkeit des Brückenbaus nicht allzu offensichtlich unterminiert, ist sie in der KMS V wenigstens sieben (vermutlich frz.) Meilen (also knapp $30 \mathrm{~km}$ ) entfernt angesiedelt. Die Saisnes - um dies vergleichend vorwegzunehmen - ziehen sich anders aus der Affäre (v. 341044 AR 4218-39 LT): unter den Augen der Sachsen und Franken durchquert ein wunderbar stattlicher Hirsch, ohne schwimmen $\mathrm{zu}$ müssen, die Rune $\mathrm{zu}$ den Franken hin, doch als diese ihn fassen wollen, verschwindet er spurlos; Sachsen wie Franken erkennen darin ein Wunder, und Karl verkündet: soeben hat

35 Hier lässt sich also die KMS V auch das uralte Motiv des «Kampfes an der Furt` nicht entgehen, wie es in breitester komparatistischer Perspektive - vom anthropologischen und folkloristischen Untergrund über lat., kelt., ags. und andere germ. zu den afrz. Texten einschließlich der Sachsenepik - kürzlich Barbieri behandelt hat; vgl. Alvaro Barbieri, Combattere al guado: realtà storica e radici antropologiche di un motivo letterario, in: L'immagine riflessa 18 (2009) 23-55. $\mathrm{Zu}$ betonen bleibt aber, dass dieses Motiv sowohl in der KMS V als auch (in transformierter Form) in den Saisnes nur eine Episode unter vielen bildet; was die ganze Handlung trägt, ist der Fluss in der Fülle seiner Aspekte, von denen die Furt nur einer ist. 
uns Jesus den Platz gezeigt, wo der Fluss am wenigsten tief und damit für den Brückenbau am besten geeignet ist! Hier werden also die beiden vorgefundenen, nahezu widersprüchlichen Einzelmotive Furt und Brücke durch Kombination erneuert. Das tat durchaus not; denn zumindest das Motiv der Hirsche oder Hirschkühe, die Kämpfern eine unsichtbare Furt offenbaren, war längst zum Cliché geworden. ${ }^{36}$

In der KMS V ist auch damit die strukturtragende Rolle des Rheins nicht erschöpft: er wird nicht nur von Franken, sondern auch im umgekehrten Sinne durchquert. Sozusagen als sein eigener Gesandter erscheint in Kap. 25-26 plötzlich Guiteclin vor Karl und beleidigt ihn: Karl sei ein Bastard, nicht Sohn Pippins, sondern eines gewissen Arnulf! Karl verteidigt sich verbal mit gebührender Heftigkeit, lässt aber, offenbar nach Gesandtenrecht, Guiteclin unbehelligt zurückkehren. Auch hier liegt Historie zugrunde. Der Spitzenahn der Karolinger/Arnulfinger ist bekanntlich Arnulf von Metz; doch Karls des Großen Urgroßvater Pippin der Mittlere hatte zu Lebzeiten seiner Frau Plektrud eine gewisse Alpheid geheiratet, was nach germanischer, nicht aber katholischer Auffassung als Friedelehe durchgehen konnte, hatte mit ihr Karl Martell gezeugt, war aber reumütig zu Plektrud zurückgekehrt und hatte nun Karl Martell so energisch von seiner Nachfolge ferngehalten, dass er, nach dem Tod seiner ehelichen Söhne, auf dem Totenbett statt Karl sogar einen Enkel im Kindesalter unter Plektruds Vorherrschaft als Hausmeier einsetzte. In der Realpolitik scheiterte Plektrud, Karl setzte sich durch. Aber nach katholischer Auffassung war er ein außereheliches und damit nicht erbberechtigtes Kind, so dass man letztlich auch den weiteren Erbgang und so die gesamte karolingische Dynastie als illegitim betrachten konnte. Die afrz. Epik spiegelt dies mehrfach, wiewohl gebrochen. Außer der Stelle in der KMS V gehört hierher in den Saisnes (v. 81 AR 78 LT) Bodels Behauptung, ein Ansëis 〈Ansegis(el)〉, Pippins [des Jüngeren] Vater, also Karls des Großen Großvater väterlicherseits, sei außerehelicher Sohn des Königs von Frankreich und der Tochter eines Kuhhirten gewesen; hier ist lediglich die doppelte Generationenfolge Pippin-Karl der Geschichte als solche nicht erkannt, so dass als Großvater nicht Karl Martell, sondern, zwei Generationen zurück, Ansegis(el), der historische Vater Pippins des Mittleren, genannt wird. ${ }^{37}$ Vor allem aber will die Sage von Karls des Großen Mutter Berthe au

36 Die lat. Stellen haben Gaston Paris (wie Anm. 12) 261, 262, 286, 360 und systematischer Pio Rajna (wie Anm. 21) 249-254 gesammelt. Durch die afrz. Epik verfolgt das Motiv Jeanne Baroin, À propos du cerf épique, in: Marche Romane 30/3-4 (1980) 5-15.

37 Später (v. 2271-91 AR 2027-44 LT) ruft ein Sachse denselben Sachverhalt (als einen Makel, der die gesamte fränkische Seite treffen soll) über den Fluss dem unerkannten Karl zu, der ihn nicht etwa leugnet, sondern nur erstaunt fragt, woher der Sachse das wisse. 
grand pied in ihrer Genesis nicht eine romantische Fabel von einer ungerecht entthronten Frau, sondern der Nachweis sein, dass Karl [Martell > der Große] entgegen allem Schein kein Bastard ist; das erkannte schon Pio Rajna, dessen dann zu Unrecht in Misskredit gefallene These ich vor Jahren mit einigen neuen Argumenten rehabilitiert $\mathrm{zu}$ haben hoffe. ${ }^{38}$

\section{c In den Saisnes}

Wie es sich für einen Karl den Großen gehört, hat Karl in den Saisnes nicht die geringste Mühe, mit seinem Heer in Köln über den Rhein zu setzen nach Saint Herbert dou Rin, dem heutigen Köln-Deutz. ${ }^{39}$ Nach nur kurzem Marsch in das Feindesland, wo das Heer sogleich mit Plünderungen beginnt (v. 1271 AR 1207 LT), wird nach einem Ritt a esperon brochant der Fluss Rune sichtbar (v. 1273 AR 1209 LT). Dieser wird nun strukturell dieselbe Rolle spielen wie der Rhein in den beiden Darstellungen der KMS. Die Erzählung ist der KMS V in den jetzt ausgedehnten Liebesszenen extrem überlegen, doch sonst in der Struktur sehr ähnlich.

Denn nachdem die Heere, durch den Fluss getrennt, zwei Jahre einander gegenübergelegen haben, entschließt sich Karl auch hier zum Bau einer Brücke (v. 2847-59 AR 2575-87 LT); vollendet wird diese aber erst nach etwa tausend Versen von AR (mit v. 3732), mehr als zweitausend Versen von LT (mit v. 4704). Auch hier werden zum Bau die Alemant und Bayern herangezogen (v. 3449 AR, in LT v. $4243 \mathrm{~s}$. auch die Lombarden und Bourguignons); auch hier desertieren speziell die Alemant und müssen zurückgeholt werden (v. 3467-3536 AR 42944353 LT). Auch hier bauen die Sachsen noch schnell ein Fort nahe dem Ausgang der Brücke und überschütten die Bauenden mit einem solchen Pfeilregen, dass Karl umplanen muss (v. 3630-65 AR 4538-78 LT). Die Szene mit dem ehernen Karl fehlt, wohl, weil Bodel die Apparate-Mode schon für abgebraucht hielt; stattdessen lässt Karl Schiffe gleich im Plural bauen zu einer Schiffsbrücke, die an der Rune wohl leichter möglich war als am Rhein (v. 3667-97 AR 4580 s. LT). Eine Elite von gut Gepanzerten - natürlich mit Balduin und Berart - setzt über und vernichtet das Fort (v. 3698-3704 AR 4588-4686 LT). Fast gleichzeitig

38 Rajna (wie Anm. 21) 199-205; Gustav Adolf Beckmann, Berthe au(x) Grand(s) Pied(s) ou plutôt les Enfances d'un ‘faux bâtard), in: Cahiers de Civilisation Médiévale 51 (2008) 313-327, passim.

39 Zum Namen: Erzbischof Heribert von Köln (sedit 999-1021) gründete 1002/03 in Deutz eine Abtei, die seine Grabstätte und bald auch Zentrum eines ihm gewidmeten Heiligenkultes wurde. 
wird endlich die Brücke fertiggestellt, und das Gesamtheer kann, wieder nach Stämmen geordnet, den Fluss überqueren, was auch hier eine Woche dauert (v. 3735-55 AR 4706-17 LT).

Bevor der Brückenbau ernsthaft begann, durchschwamm in umgekehrter Richtung auch hier Guitechin (so passim A)/Guiteclin (so passim RTL) die Rune, aber mit einer Truppe zu einem Überraschungsangriff, der dann jämmerlich scheiterte, weil Sebile die Franzosen gewarnt hatte (v. 2139-2386 AR 18992136 LT). Von den Franzosen überqueren den Fluss in ihren Herzensangelegenheiten Balduin dreimal (v. $1609 \mathrm{ff} ., 2418 \mathrm{ff}$., $3101 \mathrm{ff}$. AR $1533 \mathrm{ff} ., 2165 \mathrm{ff}$., 2817 ff. TL, ein viertes Mal in TL v. 3444 ff.) und Berart einmal (v. $2881 \mathrm{ff}$. AR 2608 ff. LT), wobei sie auf dem Rückweg in große Gefahr geraten, ferner einmal die ganze Truppe der Hurepois ${ }^{40}$ aus gereiztem Ehrgefühl (v. 2601-2772 AR 2343-2508 LT). In LT, v. 3174 ff., erlaubt sich sogar Karl ein solches Abenteuer, weil Berart ihn der Untätigkeit geziehen hatte.

Kurzum, die Rune ist in den Saisnes als Strukturachse der Erzählung mindestens ebenso unentbehrlich und ebenso ergiebig wie der Rhein in der KMS.

\section{Wo befindet sich eigentlich diese Brücke, und warum gerade dort?}

\section{a In der KMS I: als Rheinbrücke bei Wesel}

In der KMS I erfährt Karl von Vitakinds Überfall auf den Bischof von Münster in Aachen, sammelt sein Heer, bricht zur Vergeltung auf und stockt am Rhein wegen des Fehlens einer Brücke, die er dann, wie oben beschrieben, unter großen Schwierigkeiten erst nach dreieinhalb Jahren fertigstellt. Wo am Rhein befindet sie sich? Schon das Stichwort Münster sollte unsere Blicke relativ weit nach Norden lenken. Laut KMS I «überquert nun Karl mit seinem Heere die Brücke nach Vesklara(borg) hin.${ }^{41}$ Obwohl diese Stadt, wie sich zeigen wird,

40 Die große Nebenhandlung der Hurepois, in den ersten zwei Dritteln szenenweise isolierbar (insgesamt mehr als 1250 Verse), nach v. 3000 AR 2600 LT in der Haupthandlung aufgehend, würde eine eigene Darstellung lohnen, die hier nicht gegeben werden kann.

41 pa for Karlamagnus yfer bruna med her sin til Vesklaraborgar. Das til «nach, bis nach〉 kann Übersetzung eines einfachen $a$ oder vers sein. Vorwegnehmend sei schon hier festgstellt, dass ich es für eine Überinterpretation halte, wenn Paul Aebischer, La mesnie Doon de Mayence et son plus illustre représentant, Ogier le Danois, in: Mélanges de langue et de littérature médiévales offerts à Pierre Le Gentil, Paris 1973, 13-32, hier 22, aus ihm schließen will, Vesklaraborg könne nicht Wesel sein, dessen Kern nur 2,5 km vom Rheinufer entfernt ist, sondern müsse weiter östlich liegen. 
befestigt ist, harrt der dort befindliche Vitakind nicht gegen Karl aus, sondern überlässt ihre Verteidigung einem Unterführer und zieht sich zurück nach Trimonie(borg) afrz. Tremoigne «Dortmund», wo er sich, wie vorwegnehmend gesagt wird, noch drei Jahre wird halten können. ${ }^{42}$ Zwei Umstände zeigen hier, dass Vesklara(borg) eine Stadt am oder in unmittelbarer Nähe zum Rhein ist: erstens schließt der Erzähler sein «nach Vesklara(borg) hin` an das Verb «überqueren〉 an, kommt also ohne ein weiteres verbum eundi (‘und marschierte) aus, wie man es andernfalls bei seinem betulich-schrittweisen Erzählstil erwarten sollte; zweitens liegt selbst Vitakinds Rückzugsposition Dortmund nur 50-60 km vom Rhein entfernt. Roland und Olivier können mit einiger Mühe Vesklara erobern, als die Belagerten einen Ausfall wagen. Die beiden ziehen dann weiter nach Dortmund, wo vor der Stadt Karl schon wartet; er ist offenbar Vitakind nachgeeilt, hat aber inzwischen nichts erreicht und befiehlt nunmehr den beiden den Angriff. Gott wiederholt das Jericho-Wunder, Vitakind wird getötet und damit Sachsen «befreit», wie es ausdrücklich heißt; Dortmund war also Vitakinds Hauptstadt, und der Krieg ist zu Ende. Karl kann sich auf den Rückweg nach Aachen machen; dazu kann er jetzt natürlich die einfachste Route nehmen. Wie verläuft sie? Wie der Hinweg: Karl und sein Heer begeben sich zurück nach Vesklara, «wo sie die Nacht verbrachten〉. Diese Formulierung legt nahe, dass Vesklara nur einen Tagesmarsch von Dortmund entfernt ist, und zeigt überdies, dass die Route Dortmund-Vesklara-Aachen in der Realität, auch wenn sie nicht einmal annähernd eine Gerade zu sein braucht, so doch kein so großer Umweg sein kann, dass sie auch in einem Zeitalter ohne brauchbare Karten Widerspruch hervorgerufen hätte. (Im Rückblick bestätigt sich damit, was man ohnehin hätte erwarten dürfen: dass nämlich Karl schon bei seinem Aufbruch aus Aachen Vitakinds Hauptstadt Dortmund zum Ziel hatte, und es wird zudem plausibel, dass sich Vitakind von Vesklara gleich nach Dortmund, nicht nach einem kleineren, dazwischen liegenden Ort zurückzog.) Von Vesklara schickt Karl nun Gerard von Nijmegen rheinabwärts in dessen Heimatstadt, dort für Karl das Pfingstfest vorzubereiten; ${ }^{43}$ er selbst begibt sich aber

42 Der Text (der hier nur in Ms. A erhalten ist) hat an dieser ersten Stelle unsinnigerweise Triverisborg «Trier〉, nimmt das aber kurz danach zweimal richtig auf als Trimonieborg «Tremoigne, Dortmund). Dem geistlichen Übersetzer im Norden oder einem Abschreiber war die Erzbischofsstadt lat. Triveris ‘Trier` ein Begriff, während er Dortmund in der französierten Namensform zunächst nicht erkannte. Lacroix in seiner sonst sorgfältigen Übersetzung (wie Anm. 2) 136 Anm. 2 hat den Fehler bemerkt, erklärt ihn aber versehentlich falsch herum: «Tremonieborg - erreur pour Triverisborg», was die ganze Erzählung geographisch und historisch schlechthin absurd machen würde.

43 In Nijmegen ließ der historische Karl laut Einharts Vita Karoli, cap. 17, eine Pfalz egregii operis anlegen, die er seit 777 oft aufsuchte und die bis 1247 Königsgut blieb; vgl. LM (wie Anm. 14) s. v. Nijmegen. In der KMS I muss sich Karl nach Nijmegen in seine Pfalz begeben, 
zunächst zurück nach Aachen, offenbar, um dort inzwischen aufgelaufene Staatsgeschäfte $\mathrm{zu}$ erledigen. Daraus folgt, dass es von Vesklara einerseits rheinabwärts nach Nijmegen, andererseits nach Aachen keine gemeinsame Wegstrecke mehr gibt; in der Tat müssen ja schon nach dem vorher Gesagten die beiden Wege nahezu senkrecht aufeinanderstehen.

Von den ganz wenigen Orten, die hiernach geographisch in Frage kommen, trägt nur Wesel einen Namen, der an Vesklara anklingt, worauf schon der Niederrheiner Theodor Frings knapp hinwies. Da Frings' geographische Erkenntnis später von Aebischer m. E. zu Unrecht bestritten wurde, ${ }^{44}$ sei sie hier auch auf eine realhistorische Basis gestellt. Wesel liegt fast am Rhein (genauer gesagt: der Ortskern liegt 2,5 km vom Rheinufer entfernt) und zugleich am Nordufer der dort mündenden Lippe; es kann deshalb einen sehr interessanten Anspruch anmelden. Denn jeweils auf dem Weg in den Sachsenkrieg überquerte den Rhein schon Karl Martell 738 an der Lippemündung (Fredegar-Fortsetzer cap. 19, SS.mer. 2.177), dann Karl der Große nach den Reichsannalen zumindest 779, 784 und 799 genauer bei einem Lippeham an der Lippemündung. ${ }^{45}$ Dieser Name ist später - außer bei Ausschreibern der Annalenstellen - nicht mehr belegt. Doch da Karl der Große damals jeweils ins nördliche Sachsen wollte, lag offensichtlich Lippeham wie Wesel auf der Nordseite der Lippe; denn es ging ja darum, sich eine weitere Flussüberquerung $\mathrm{zu}$ ersparen. ${ }^{46}$ Hier ist -ham nie-

weil anschließend erzählt werden soll, wie dort zu seiner Überraschung auf dem Rhein in einem von einem Schwan gezogenen Nachen ein fremder Ritter erscheint, eben der «Schwanenritter, der dann in Karls Dienste tritt; die Nijmeger Tradition der Schwanenritter-Sage wird ausführlicher erzählt von Philippe Mouskés (v. 16025 ff.). (Genau genommen liegt Nijmegen dicht westlich der Gabelung des Rheins an der breiteren Waal, nicht am schmaleren Nederrijn; doch niemand wird von einem mittelalterlichen Autor eine solche pedantische Genauigkeit verlangen.) Dem nordischen Übersetzer passiert hier wiederum ein kleines Missgeschick: er vergisst mitzuteilen, dass sich Karl inzwischen, wie geplant, von Aachen nach Nijmegen begeben hat, so dass es so aussehen könnte, als blicke Karl in Aachen auf den Rhein.

44 Frings, Theodor, Rez. zu Aebischer, Paul, Textes norrois et littérature française du Moyen Âge, I, Recherches sur les traditions épiques antérieures à la Chanson de Roland d'après les données de la première branche de la Karlamagnús saga (Genève 1954), in: ZrP 73 (1957) 175182, hier 177; Aebischer (wie Anm. 41), $22 \mathrm{f}$.

45 Und noch einmal a. 810 auf dem Weg in den drohenden Dänenkrieg.

$46 \mathrm{Zu}$ a. 799 darf man den Text der Reichsannalen vermutlich nicht so weit pressen, als wollten sie Lippeham auf dem linken Rheinufer gegenüber der Lippemündung situieren. Doch selbst wenn es wider Erwarten dort gelegen haben sollte, tangiert das die obigen Darlegungen nicht, da ja Karl auch in diesem Fall das östliche Rheinufer bei Wesel betreten hätte. Die Lage der Lippemündung wiederum kann sich seit damals, wenn überhaupt, nur noch so geringfügig verändert haben, dass dies hier keine Rolle spielt. - Dass der Weg von Aachen über Wesel nach Dortmund immer noch in Lippeham/Wesel einen für heutige Verhältnisse beträchtlichen Knick hat, konnte ein mittelalterlicher, noch dazu romanophoner Autor (ohne Deutschland- 
derdt. ham «Winkel (insbesondere des Landes an einem Wasser), Bucht, Anlegeplatz der Schiffe»; ${ }^{47}$ Lippeham 〈Lippe-[Mündungs-]Bucht〉 ist also eine naturgeographische Bezeichnung, nicht notwendigerweise der Name einer Siedlung. Die nächstgelegene Siedlung ist vielmehr Wesel, dessen frühe Geschichte sich wie folgt liest: ${ }^{48}$ «Der zum Reichsgut [! G.A.B.] gehörende Salhof Wisele wird im 8. Jh. als zum Kloster Echternach gehörig erwähnt, um 1100 ist eine Kaufmannssiedlung nachweisbar. Schon vor der Stadterhebung 1241 [...] besaß Wesel eine eigene Verwaltung mit dem Bürgermeister an der Spitze des Schöffenkollegiums. [...] Dank zahlreicher Privilegien wurde Wesel ein bevorzugter Standort für Kaufleute. Dafür sprechen auch die frühe Anwesenheit von Juden (ab 1266) und Lombarden (ab 1301) sowie die Weseler Münze.» Wenn die Karolinger dort im 8. Jh. zunächst selbst einen Wirtschaftshof unterhielten, so war dieser offensichtlich für den benachbarten Landeplatz der humangeographische Support, wo z. B. auch die Könige auf Fahrten nach und von Sachsen übernachten konnten; in ähnlicher Perspektive wurde er dann dem fernen Kloster Echternach übergeben, das rechtlich ja ein karolingisches Eigenkloster war und für das er als Zwischenstation zu seinen beträchtlichen Besitzungen in den Niederlanden wertvoll sein musste. ${ }^{49}$ Wesel war zwar knapp noch stammesfränkisch, doch weniger als $20 \mathrm{~km}$ weiter östlich und nordöstlich begann das damalige Sachsen. Da kann sich sehr leicht eine regionale Erinnerung gehalten haben, in der lediglich Wesel an die Stelle des unüblich gewordenen Lippeham getreten war, als die Stelle, wo der große Karl zum Sachsenkrieg überzusetzen pflegte.

Nun ist die verlorene afrz. Grundlage der KMS I, in der Forschung als Vie de Charlemagne bekannt, zwischen 1216 und 1238 von einem Bischof von Lüttich in Auftrag gegeben worden, und ihr Kompilator war ein fleißiger Sammler, der in sein Werk gleich hinter den Sachsenkrieg die Schwanenritter-Sage in ihrer niederrheinischen Lokalisierung (um Nijmegen) aufnahm (ed. Unger cap. 48, ed. Loth cap. A 45). ${ }^{50}$ Wesel, von Lüttich in einem verkehrsgeographisch gut erschlossenen Gebiet in der Luftlinie nur $140 \mathrm{~km}$ entfernt, stand damals als markanter Handelsplatz am Rhein zweifellos auch mit der Wallonie durch wallonische und eigene Kaufleute in ständiger Beziehung. Diese dürften, wie die

karte!) kaum erkennen; der historische Karl nahm fast den gleichen Knick auf sich, als er laut den Reichsannalen 799 von Aachen über Lippeham nach Paderborn zog.

47 Vgl. z. B. Ernst Wilhelm Förstemann, Altdeutsches Namenbuch, II, Orts- und sonstige geographische Namen, ${ }^{3}$ Bonn 1913, s. v. Hamm, und Hermann Jellinghaus, Die westfälischen Ortsnamen nach ihren Grundwörtern, Kiel 1896, 40.

48 LM (wie Anm. 14) s. v. Wesel.

49 Vgl. LM (wie Anm. 14) s. v. Echternach.

50 Vgl. Beckmann (wie Anm. 22), speziell 7-38 und 195-223. 
niederrheinische Schwanenritter-Fassung, so auch die niederrheinische Sachsenkriegs-Fassung samt der Weseler Reminiszenz, dass Karl immer hier bei uns) zum Sachsenkrieg durchzog, dem Kompilator vermittelt haben. ${ }^{51}$

\section{b In der KMS V: als Rheinbrücke bei Worms}

Eine zentrale Rolle spielt der Rhein in KMS V lange, bevor dort der Plan eines Brückenbaus aufkommt. Kaum in Eile aus Spanien nach Köln zurückgekehrt, das die Sachsen wieder geräumt haben, hat Karl den leichtsinnigen Einfall, als Beginn des Feldzuges mit einem Gefolge von nur eintausend Mann über den Rhein zu setzen, um Guiteclin durch eine Jagdpartie in dessen Reich zu demütigen - wie denn ja auch der historische Karl ein leidenschaftlicher Jäger war, selbst noch kurz vor seinem Tode (vgl. Einharts Vita Karoli 22 und 30). Die Franken werden durch dreißigtausend Sachsen unter Guiteclin vom Rhein abgeschnitten, können sich aber in eine verlassene Burg zurückziehen. Karl schickt einen mutigen Boten durch die feindlichen Linien mit einem Hilferuf an Roland. Der erobert noch schnell im Sturmangriff Nobles, hier ohne Jericho-Wunder, und eilt zunächst nach Köln, dann (offenbar in der Nähe) zu einem Treffen mit Turpin. Der Erzbischof bringt ihn dazu, nicht sogleich den Rhein zu überschreiten, sondern als Entlastung für Karl zunächst eine der wichtigsten Städte in Guiteclins Reich zu erobern, nämlich Garmasie (A)/Garmaise (B,b) 〈Worms $\rangle^{52}$

51 Die lautliche Entwicklung bedarf des Kommentars. Germ. $w$-ist im Wall. erhalten (nicht $>\mathrm{gu}$-), im Altnord. vor hellem Vokal von vornherein $v$ - (wohl schon labiodental). Der Name Wisele/Wesele wurde offenbar im benachbarten wall. Sprachgebiet schon bekannt, als sein -snoch stimmlos war; nach Frings (wie Anm. 44) 177 entstand dann das /k/ als rom. Gleitlaut (vgl. slav- > afrz. esclaf, esclave, Esclers/Esclavons, germ. slag > afrz. esclou, ahd. slahta/slatha $>$ afrz. esclate ‘Geschlecht, germ. slit(j)an > afrz. escli(c)ier). Vesklara kann zudem Kreuzung mit einem Vauclere (< ${ }^{\star}$ Valclere) aufweisen, das im Doon de Mayence (bzw. seiner verlorenen Vorform) die (von Mainz aus) zuerst erreichte Stadt in Sachsen, aber ein offensichtlicher Phantasieort ist (inmitten großer Wälder gelegen, wird es gerade von den Dänen belagert; zudem durchsichtige Etymologie).- Aebischer, der 1954 für Vesklara Wetzlar und Fritzlar, beide im (frankisierten) Hessen, ins Gespräch gebracht hatte, nahm dies 1973 wieder zurück; vgl. Aebischer (wie Anm. 41) 23. In der Tat passen beide schon geographisch ausnehmend schlecht in die Erzählung. Zudem ist Wetzlar zu jung (Existenz im 10. und 11. Jh. nur zu erschließen, Erstbeleg 1145). Fritzlar macht eine phonetische Zusatzannahme nötig (anderweitig unbelegte Schwunddissimilation des ersten $-r$-); dadurch, dass es 774 von den Sachsen während ihres Überfalls eingeäschert wurde (LM [wie Anm. 14] s. v. Fritzlar), wird es für unsere Zwecke nicht geeigneter.

52 Garmaise/Guarmaise/Gormaise (< früh-mlat. Warmatia/Wormatia) heißt Worms auch im Garin le Loherenc, Doon de Nanteuil, Doon de la Roche, Roland-V4, Chevalier au Cygne, Roman de la Violette und Gui de Warewic; vgl. André Moisan, Répertoire des noms propres de person- 
(das ja in der Tat auf dem linken Rheinufer liegt); gelinge das, könne man damit Karl durchgreifend helfen. Roland und Olivier erobern Worms, und Guiteclin zieht sich mit allem, was er besitzt, zurück auf das rechte Rheinufer. Karls Bote findet zu seinem Herrn in die belagerte Burg zurück und kann ihm die Sachlage melden; Karl bricht mit seiner Truppe erfolgreich aus und erreicht - anscheinend ohne längeren Marsch - Roland und das Hauptheer (Kap. 2-16). Die Burg liegt also zwar rechts des Rheins, doch nahe bei Worms. Dass Karl zur Vereinigung mit Roland den Rhein überquert haben muss, wird nicht gesagt, ergibt sich aber sogleich. Denn jetzt ist ja erst Karls Eskapade zu Ende, und erstmalig steht jetzt das Hauptheer vor der Aufgabe, zur Verfolgung Guiteclins den Rhein zu überschreiten; dazu bedarf es eben einer Brücke. Noch in Kap. 16 entschließt sich Karl zu ihrem Bau, welcher dann, wie oben beschrieben, die an Wechselfällen reichen Kapitel 16-36 strukturiert.

Wie eingangs erwähnt, erzählt schon der PT in seinem Anhangskapitel 33 essenziell dasselbe: Karl der Große sitzt während des Sachsenkriegs fest in einer Burg bei Worms und ruft seinen Neffen von (Gre-)Noble[s] zu Hilfe.

Nun spielen sich diese Vorgänge ja nicht in fernen Landen, sondern sozusagen vor Frankreichs Tür ab. Dass da das linksrheinische, stammesmäßig altfränkische Worms überhaupt in den Sachsenkrieg gerät, dass ganz in der Nähe Karl von dem Sachsenführer in die Enge getrieben wird, ja dass Worms - jedenfalls in der KMS V - sogar dem Sachsenführer gehört, das wirkt zunächst bizarr, überschreitet das Maß an geographisch-historischer Toleranz, das wir einem Zeitalter ohne brauchbare Europa-Karten und mit beschränkten Bibliotheken zuzugestehen bereit sind. Und immer wenn dergleichen vorliegt, tut die Epenforschung gut daran, nach einer speziellen Ursache zu suchen.

Das hat hier vor mir, aber wie ich glaube, mit inakzeptablem Ergebnis schon de Mandach versucht. ${ }^{53}$ Ihm zufolge steht hinter Warmatia/Garmaise/ Gormaise das span. Gormaz am Nordufer des oberen Duero. Dieses war von den

nes et de lieux cités dans les Chansons de geste françaises et les œuvres étrangères dérivées, 5 Bde. in 3 〈tomes〉, Genève 1986, und Léopold Flutre, Table des noms propres avec toutes leurs variantes figurant dans les romans du Moyen Âge écrits en français ou en provençal et actuellement publiés ou analysés, Poitiers 1962, beide s. v. Früh-mlat. Warmatia/Wormatia seinerseits ist Latinisierung der (anscheinend unbelegten) ahd. Vorstufe von mhd. Wormez, die durch zweifache Dissimilation (und zweite Lautverschiebung) aus (kelt.-) lat. Borbeto(magus) entstanden war.

53 Mandach, André de, La Prise de Nobles et de Garmaise par Roland, in: Essor et fortune de la chanson de geste dans l'Europe et l'Orient latin, Actes du IX ${ }^{\mathrm{e}}$ Congrès International de la Société Rencesvals, Padoue-Venise, 29 août-4 septembre 1982, 2 Bde., Modena 1984, 717-728, hier 721-725. Ders., L'Entrée d'Espagne: six auteurs en quête d'un personnage, in: Studi Medievali 30 (1989), 163-208, hier Abschnitt 7. 
Muslimen um 960 zur größten und stärksten Festung von al-Andalus ausgebaut worden, fiel aber trotzdem um 1060 an Ferdinand I. von León (Chronica Najerensis 3.1.19, 3.8.9) in einem Feldzug, bei dem die Geschichte von französischen Helfern ebenso wenig weiß wie bei irgend einer anderen Unternehmung Ferdinands. Bei de Mandach ist diese Idee verknüpft mit der ebenso unhaltbaren, hinter dem Noples/Nobles des Rolandsliedes stehe der $7 \mathrm{~km}$ von der Festung Gormaz entfernte Ort Navas de Palos (so noch im Cantar de mio Cid v. 401!), später Navapalos. Doch die bloße Ähnlichkeit der Namen Gormaz und Ga/ormaise scheint mir bei weitem nicht auszureichen, einen räumlichen Transfer über $1600 \mathrm{~km}$ und einen personellen (den bisher einzigen!) von Ferdinand auf Karl anzunehmen, zumal erstens nichts darauf hindeutet, dass Ferdinand bei Gormaz in Gefahr geriet, somit eine narrative Ähnlichkeit nicht auszumachen ist, und zweitens Ga/ormaise und Noples/Nobles in aller Epik nicht benachbart sind, sondern notorisch weit auseinanderliegen.

Ich möchte der Erklärung de Mandachs eine eigene entgegenstellen, bei der von vornherein der Frankenkönig Karl [der Große] ein Frankenkönig Karl, Warmatia/Garmaise eben Worms-am-Rhein und der Sachsenführer ein Sachse ist, der Worms als Teil seines Reiches ansieht und Karl ebendeshalb in eine missliche Situation bringt - kurzum eine Erklärung, bei der diese Determinanten der Erzählung von vornherein das sind, was sie auch in der Erzählung sind.

Dafür müssen wir uns ein wenig die Vorgeschichte der relevanten Situation ins Gedächtnis zurückrufen. Bei der ersten Teilung von Karls des Großen Reich 840 erhielt das Ostreich unter Ludwig, später «der Deutsche〉 genannt, linksrheinisch nur das kleine Gebiet um Mainz-Worms-Speyer, und auch das nur als minimales altfränkisches Gegengewicht gegen die Überzahl der stammesfremdem Alemannen, Bayern, Thüringer und Sachsen und der erst oberflächlich frankisierten Hessen. Hingegen gehörten Köln, Koblenz, Trier und Straßburg zum Mittelreich, d. h. zu der in ihrem nordalpinen Teil bald sogenannten Lotharingia, die 880 an das Ostreich fiel. Doch 911 starb der letzte ostfränkische Karolinger, und schon 913 konnte der Westfrankenkönig Karl III., von Späteren sehr zu Unrecht «der Einfältige〉 genannt, die Lotharingia in das Westreich eingliedern, z. T. dank der karolingischen Sympathien des lotharingischen Adels. In dieser Situation musste in Frankreich die antike Lehre, Galliens Ostgrenze sei der Rhein (wie sie z. B. Cäsars Bellum Gallicum 1.1 s. und Plinius' Naturalis Historia 4.105 zugrunde liegt), nach langer Latenz neu aufleben, doch jetzt mit Francia als modernem Synonym von Gallia; denn zur vollen Rheingrenze fehlte nur noch der Besitz von Mainz-Worms-Speyer. A. 919 wurde der Sachsenherzog Heinrich I. zum König des ostfränkischen Reichs gewählt. Heinrich musste damals zunächst sein Königtum und Königreich sichern, konnte also noch nicht an eine Reannexion Lotharingiens denken. Aber umgekehrt konnte auch Karl 
noch keinen Sachsenkrieg in der Art seines großen Ahnen wagen, erkannte vielmehr Heinrich als princeps, vielleicht sogar rex, transrhenensis an. Die beiden Positionen unterschieden sich somit nur in Bezug auf Mainz-Worms-Speyer.

In einer solchen Lage ist ein fait accompli viel wert. Damit sind wir bei der uns interessierenden Situation: 920 erschien Karl unvermutet mit einem Heer bei Worms. Der auf westfränkischer Seite maßgebende zeitgenössische Historiker Flodoard spricht von [rex Karolus] qui tunc morabatur in pago Warmacensi contra Heinricum principem Transrhenensem. ${ }^{54}$ Doch Karl musste weichen, wie die Folgeereignisse zeigen. Auf ostfränkischer Seite konnte man sogar von Karls Feigheit sprechen; die um 967 geschriebene Continuatio Treverensis von Reginos Chronicon sagt von Karl, dass er vom Elsass aus partes illas Franciae iuxta Rhenum usque Mogontiam sibi usurpaturus usque Paternisheim villam (Pfeddersheim, $6 \mathrm{~km}$ westl. Worms) iuxta Wormaciae [urbem] hostiliter pervenit. Unde fidelibus Heinrici Wormaciae coadunatis aliter quam decuerat regem, aufugit. ${ }^{55}$ Ähnliches schwingt mit auch bei Widukind von Corvey: Ob quod [scil. der Ansprüche Karls] Heinricus rex movit castra sua contra Karolum eiusque saepius fudit exercitum, iuvitque virum fortem fortuna. ${ }^{56}$ Gleichzeitig mit Karls Niederlage(n?) formierte sich in Paris gegen ihn der Aufstand, der drei Jahre später mit seiner lebenslangen Einkerkerung endete - und Heinrich die Reannexion der Lotharingia ermöglichte. ${ }^{57}$

In der Nähe des von «dem〉 Sachsen-par-excellence gehaltenen Worms am Rhein gerät also 〈der Frankenkönig Karl〉 eben durch diesen Sachsen in Not. ${ }^{58}$ Das ist zu viel Übereinstimmung mit der KMS V, um Zufall zu sein, zumal Ähnliches zu keiner anderen Zeit geschehen ist. Doch - man braucht es kaum hinzuzufügen - anders als die Realität hatte die Epik einen deus ex machina, der aus Karls Niederlage einen Sieg machen konnte: er hieß Roland.

Aber, so könnte man einwenden, musste das Ereignis von 920 nicht bald im Meer der folgenden Ereignisse untergehen? Keineswegs; denn als erster Sieg

54 Flodoard von Reims, Annales, zu 920, ed. Philippe Lauer, Paris 1905, p. 4; ähnlich seine Historia Remensis ecclesiae 4.16, ed. Martina Stratmann, Hannover 1998 (MGH SS. 36), 408.

55 Reginonis abbatis Prumiensis Chronicon cum continuatione Trevirensi, ed. Fridericus Kurze, Hannover 1890 (MGH SS.schol. 50), 157. Die Continuatio setzt dies unter a. 923 statt a. 920, was sachlich ausgeschlossen ist; in der Tat unterlaufen dem Autor für die Jahre 919-936 eine Reihe von Fehldatierungen bis zu vier Jahren, vgl. die Edition.

56 Widukindi monachi Corbeiensis Rerum gestarum Saxonicarum libri tres, ed. Paul Hirsch, Hannover 1935 (MGH SS.schol. 60), 1.30, p. 42, geschrieben a. 967-968.

57 Vgl. im LM (wie Anm. 14) K. F. Werners Artikel Bonn, Vertrag von.

58 Zwar begann auch Karl der Große nach dem Bericht der Reichsannalen seinen ersten Sachsenkrieg 772 von Worms aus mit einem Zug durch Hessen zur Eresburg (vgl. weiter unten im Haupttext), doch natürlich ohne dass die Sachsen je Worms oder dessen Umgebung erreicht hätten. 
eines Sachsen über einen Frankenkönig seit mehr als drei Jahrhunderten war es, mit einem Terminus Grégoires gesagt, ein premier choc, der Paukenschlag, der eben das ottonische, dann das salische Jahrhundert und damit das Zeitalter der permanenten Demütigung Frankreichs durch Deutschland einleitete.

Doch nichts ist endgültig verloren, wo nicht die Hoffnung verloren ist. In diesen Jahrhunderten hatte sie in Fankreich einen Namen, dem Karl soeben neues Leben eingehaucht hatte: die Rheingrenze. A. 939, 973, 978, 985, 102426, 1037, 1044, 1046-50 - in der realen Politik gab es in jeder Generation Ereignisse, bei denen sie kurze Zeit zum Greifen nah erschien; ${ }^{59}$ noch 1056 warf Heinrich I. von Frankreich Kaiser Heinrich III. bei einem persönlichen Treffen mit bitteren Worten vor, dessen Vorgänger hätten partem magnam regni Francorum - also Lotharingien - durch List okkupiert. ${ }^{60}$ Ebenso bestimmte die Rheingrenze das Bewusstsein der Historiographen: Mitte des 10. Jh. nennt Flodoard von Reims (Hist. Rem. Eccl. 4.5) rückblickend sogar Kaiser Arnulf († 899) rex Transrhenensis, Ende des 10. Jh. Richer von Reims den Deutschen Heinrich I. († 936) nur Heinricus Transrhenensis und Saxoniae dux (erste Redaktion 1.20, 35 s.) bzw. Saxonię rex (zweite Redaktion 2.18), schließlich noch um 1040 der im Herzogtum Burgund, also im regnum Franciae, schreibende Raoul Glaber (1.4, 3.1) das Reich Heinrichs I. und Heinrichs II. († 1024) und das Land, in dem Bamberg [!] liegt, «Sachsen`. Nun waren aber die Nachfolger dieser Sachsen, die Salierkaiser, ausgerechnet Wormser par excellence, ein ganzes Jahrhundert lang (1024-1125) bis nur etwa zwei Jahrzehnte vor dem Augenblick, wo Worms im PT auftaucht. Das half, den Namen Garmaise, das Wissen um die linksrheinische Lage der Stadt und eine vage Erinnerung an 920 lebendig zu erhalten; doch führte es auch dazu, dass in der KMS V die Stadt, obwohl linksrheinisch, zu Guiteclins «Sachsen〉 (in der Realität: zum Ostreich) gehörte.

Das Wichtigere, Allgemeinere, war selbstverständlich das Postulat der Rheingrenze. Aber eine Grenze hat zwei Seiten: die Rheingrenze zu postulieren, hieß eben, anzuerkennen, dass jenseits davon das Land des Anderen, de l'Autre mit Majuskel, begann. In der Sachsenepik konnten das nur die Sachsen sein. Indem nun, von der hybriden Stellung von Worms abgesehen, die beiden KMSErzählungen - und zweifellos, wie der PT zeigt, schon ihre französischen Vorstufen - das populäre Rheingrenze-Postulat des 10.-12. Jahrhunderts in die Zeit Karls des Großen zurückprojizierten, ergab sich jenes durchaus unhistorische Grundfaktum beider Erzählungen, das wir bisher unhinterfragt akzeptiert

59 Die Ereignisse sind z. B. in den jeweiligen Jahrbüchern und Herrscherbiographien leicht aufzufinden, so dass ich auf Einzelnachweise verzichten kann.

60 So Lamperti monachi Hersfeldensis opera, ed. Oswald Holder-Egger, Hannover 1894 (MGH SS.schol. 38) $68 \mathrm{zu}$ a. 1056. 
haben: dass nämlich für Karl und sein Heer die Überschreitung schon des Rheins zum Problem epischer Größe werden konnte. Dass der Rin hier sekundär, als lectio facilior, für einen anderen Fluss, die Rune, eingetreten ist, zeigen die Saisnes, die die ältere Erzählung bewahrt haben. Wenden wir uns ihnen zu!

\section{c In den Saisnes: als Ruhrbrücke nahe Dortmund; das Problem der beiden Rune}

In den Saisnes beginnt unmittelbar, nachdem die Franzosen endlich die Rune überschreiten konnten, die Entscheidungsschlacht; sie ist sehr blutig, weil Guitechin unerwartet Verstärkungen erhält (AR v. 3733-4097 LT 4705-5277), geht dann aber über in das oben behandelte Duell zwischen Karl und Guitechin mit Guitechins Tod. Die Sachsen fliehen; die Franzosen verfolgen und dezimieren sie zwar, kehren dann aber zu Karl zurück (AR v. $4129 \sim$ LT v. 5372). In AR folgt nun das Verspaar (4130 s.): Charles vint a Tremoigne o son riche barné, / La nuit jut l'empereres el gran palais pavé; Dortmund ist also ohne weiteren Schwertstreich gefallen, und Karl kann mit dem Zusammengeben der beiden neuen Paare das erwartete dénouement der Handlung vollenden. In LT finden die Franzosen Sebile und Helissent im von den Männern verlassenen Feldlager Guiteclins vor, und schon hier kann Karl die Paare vereinen; Sebile fordert dann von außen Tremoigne zur Kapitulation auf, die auch erfolgt (v. 5388-5583). In beiden Fassungen ist der Weg von der Rune nach Tremoigne offenbar kurz, kürzer noch, als es der Marsch von Saint Herbert dou Rin, also (Köln-)Deutz, über die fränkisch-sächsische Grenze zur Rune war.

Nun beträgt die Entfernung von Köln-Deutz zur Ruhr bei der Hohensyburg 78, von dort zum Stadtkern von Dortmund weitere 14 Straßenkilometer, das Ganze ist eine fast gerade Linie. (Seit 1928 ist die Hohensyburg sogar in Dortmund eingemeindet, und das Stadtgebiet beginnt an der Ruhr.) Dass unter diesen Umständen die Rune die Ruhr ist, hat schon 1893 Oscar Schultz[-Gora] erkannt. ${ }^{61}$ Es liegt eine simple Dissimilation des zweiten - $r->-n$ - gegen das erste $-r$ - vor (wobei afrz. $-r$ - damals mit Sicherheit noch vorderes $-r$ - war, also dem $-n$ artikulatorisch nahestand).

Schultz beeinträchtigt allerdings diese seine Erkenntnis, indem er ihr eine verfehlte Behauptung logisch vorschaltet: der Name habe ursprünglich wohl irgend ein anderes Gewässer bezeichnet, sei dann zu einem typisch epischen Gewässernamen geworden, so dass er in den Saisnes «auch» die namensähnliche Ruhr bezeichnen konnte. $\mathrm{Zu}$ einer so verkrampften Idee kommt er nur,

61 Oscar Schultz[-Gora], Zum Guiteclin, in: ASNS 91 (1893) 247-250. 
weil es in der afrz. Epik noch einen zweiten Fluss Rune gibt: im Roland-Ms. V4 (v. 288 ed. Beretta) sagt der aus Karls Lager nach Saragossa aufbrechende Ganelon zu seinem Pferd: Vu passarì la grant aigua de Runa; und im PT (cap. 11) gelangt Karls großes Heer nach Pampilonia und bedeckt dort die Erde a flumine Runae bis zu einem drei frz. Meilen entfernten Berg. Schon kurz nach Schultz' Aufsatz konnte Antoine Thomas ${ }^{62}$ diese zweite Runa an Hand mehrerer in Spanien geschriebener Texte identifizieren als einen zweiten Namen des Arga, des Flusses, der durch Pamplona fließt; übrigens gibt es heute - ich weiß nicht, seit wann - in Pamplona zwischen dem Arga-Ufer und der Calle del Río Arga, gegenüber dem alten «Franken`viertel San Cernín, einen Parque del Runa. Es liegt also innerhalb des Afrz. einfach eine Homonymie zweier Flussnamen vor. Thomas möchte allerdings zwischen ihnen den Rest einer Verbindung retten, indem er annimmt, Rura > Rune sei unter dem Einfluss des span. Flussnamens erfolgt; aber auch das scheint mir bei einer simplen Dissimilation unnötig.

Thomas hat nun zwar die zweite Runa als den Arga identifiziert, doch nicht erklärt, weshalb der Arga einige wenige Male unter diesem ganz anderen Namen erscheint; solange das nicht geschieht, könnten wieder Zweifel an der Identifizierung aufkommen. Ich schlage deshalb, von Thomas' Belegen ausgehend, die folgende Erklärung vor. Runa erscheint erstens im PT, also im Munde eines Galloromanen (vermutlich Poiteviners); zweitens kurz nach 1276 bei einem weiteren Galloromanen, dem Tolosaner Guilhem Anelier, in seiner Histoire de la Guerre de Navarre (v. 3676 ed. F. Michel); drittens im frühen 14. Jh. bei dem franko-it. Redaktor des Roland-V4, der es am ehesten aus dem schon europaweit verbreiteten PT hatte; viertens 1406 beim Verkauf einer Liegenschaft der cofradía de San Cerní in jenem kurz nach 1100 entstandenen 〈Franken〉viertel der Stadt, das lange Zeit für die Ansiedlung von Navarresen gesperrt war, ${ }^{63}$ im 13. Jh. sogar den von Anelier beschriebenen Bürgerkrieg gegen die einheimischen Stadtviertel führte und dessen dominierende südfrz. Familien erst danach allmählich in der lokalen Aristokratie aufgingen. Und schließlich erscheint Runa fünftens in einer Aufzählung wasserreicher Flüsse in den sogenannten Fueros de Sobrarbe; Letztere sind laut Lalinde Abadía ${ }^{64}$ eine Gruppe «fiktiver Verfügungen [...], die die Macht des Königs einschränken [...] sollten», letztlich

62 Antoine Thomas, La rivière de Rune dans l'épopée française, in: Romania 23 (1894) 146148.

63 Nämlich durch Urkunde des Alfonso el Batallador von September 1129, ediert bei José Ángel Lema Pueyo, Colección diplomatica de Alfonso de Aragón y Pamplona (1104-1124), San Sebastián/Donostia 1990, Nr. 211.

64 LM (wie Anm. 14) s.v. Fueros, III; genauer Jesús Lalinde Abadía, Los fueros de Aragón, Zaragoza 1976, 95-99. 
also von Fälschungen, und bilden somit ein Sonderproblem. ${ }^{65}$ Aber selbst ein Belegverhältnis von 4:1 könnte kaum Zufall sein: Runa statt Arga ist ein essenziell gallorom. Name. Nun klingt Runa an Iruña an, den bask. Name von Pamplona. ${ }^{66}$ Und während der Nexus 〈Fluss〉 (also z. B. frz. rivière, okz. aigua, span. río) $+d e+$ Eigenname in der Iberoromania in der Regel possessiv ist (z. B. el río de Pamplona), ist er in der Galloromania entweder possessiv (la rivière de Pampelune) oder (schon afrz. und aokz.) ${ }^{67}$ identifizierend (so l'aigua de Runa im Roland-V4 oder la rivière de Rune im Titel von Thomas' Aufsatz, contra span. appositionelles el río Arga). Wo also ein Iberoromane von dem río de Iruña sprach, konnte ein Galloromane das missverstehen als «der Fluss Iruña oder, wenn wir ihm die nicht seltene Metanalyse von $d(e)+$ hellem Folgevokal und eine weitere kleine lautliche Ungenauigkeit zubilligen, als «der Fluss Runa〉. Stand ein solcher Irrtum am Anfang, so dürften die Galloromanen von San Cernín schon aus anti-navarresischem Geist lange an diesem Namen für 〈ihren〉 Fluss festgehalten haben.

Wenn so die zweite Rune als Unsicherheitsfaktor ausfällt, steht damit die Bedeutung «Ruhr für die erste Rune umso eindeutiger fest.

65 Da nach heute überwiegender Forschungsmeinung weniger ein 〈Text〉 als ein mito, ein ideologisches Konstrukt, vorliegt («en Aragón, Leyes antes [de] Reyes`), das sich im 13. Jh. auszukristallisieren begann und im 16. Jh. den Höhepunkt seiner Brisanz erreichte, ist die Frage nach einer authentischen Textform wohl schon in sich elusiv. Doch ist immerhin auffällig, weil aus dem Fälschungszweck nicht bündig ableitbar, dass schon in den frühen Erscheinungsformen dieses «Mythos` behauptet wird, die Fueros de Sobrarbe seien zwar von anti-islamischen Kämpfern der ersten Stunde in Sobrarbe verfasst worden, aber mit redaktioneller Hilfe von «ombarden und Franzosen>. Hat das einen realen Kern, so kann Runa statt Arga auch hier auf einen Galloromanen zurückgehen; ist es pure Fiktion, so können deren Urheber mit Runa bewusst ein gallorom. klingendes Sprachelement haben einfließen lassen.

66 Auf heutigen Karten liest man zwar manchmal die Variante Iruñea (= Iruña + postpositiver Artikel - $a$, vgl. Luis Michelena, Fonética Histórica Vasca, Donostia/San Sebastián 1980, 114 s.); die historisch überkommene Form ist aber Iruña. Nur sie erscheint im Diccionario de Antigüedades del Reino de Navarra von Yanguas y Miranda s. v. Pamplona (als Iruina mit $<$ in $>\sim / \tilde{n} /$ schon in der in Anm. 63 erwähnten Urkunde des Alfonso el Batallador von 1129), im LM (wie Anm. 14) s. v. Pamplona sowie in einem Brief (vom 15. 7. 1969) von Luís Michelena, dem unvergessenen Mitbegründer der modernen baskischen Sprachgeschichtsforschung, worin er mir den Namen ausführlich erklärte.

67 Nämlich als Erbe eines spätlat. Genetivs, vgl. Ernst Gamillscheg, Historische französische Syntax, Tübingen 1957, 109. 


\section{Tremoigne «Dortmund}

\section{a Inhaltlich}

Trimonia-/Tremonieborg bzw. Tremoigne 〈Dortmund〉 ist die einzige sächsische Stadt, die in allen drei Texten - der KMS I, der KMS V und den Saisnes - vorkommt. In der KMS V 51 ist sie immerhin die Heimat von Guiteclins Fahnenträger, in den beiden anderen Texten Vitakinds/Guitechins Hauptstadt, deren Fall den Krieg beendet; ${ }^{68}$ Letzteres entspricht mehr der sonstigen Erzähllogik der afrz. Epen.

Denn während die deutschen Könige und Kaiser bis über Barbarossa hinaus 〈itinerante〉 Herrscher blieben, stärkte in Frankreich gerade die anfängliche Beschränkung der Kapetinger auf eine relativ kleine Domäne die Hauptstadtfunktion von Paris. Aus der Bedrohung, die Paris 886 und 978 auszuhalten hatte, gingen die Stadt und ihre Verteidiger in ihrem Ansehen gestärkt hervor. Wo die Behauptung der eigenen Hauptstadt als Unterpfand der Zukunft galt, konnte komplementär erst die Eroberung der feindlichen Hauptstadt Erweis eines gelungenen Feldzugs sein: schon im Roland muss Saragossa Hauptstadt von ganz Spanien sein und schließlich von Karl eingenommen werden, beides gegen die Geschichte. Im Stammessachsen des realen Widukind gab es keine Städte, doch der Widukind der französischen Epik brauchte eine Hauptstadt, deren Fall seinem Fall das gebührende Echo verlieh.

Was prädestinierte gerade Dortmund zu dieser Rolle? ${ }^{69}$ Dortmund profitierte vor allem vom Verkehr. Die beiden wichtigsten Wege schon durch das heidnische Sachsen waren der Hellweg vom Rhein bei Duisburg zur Weser bei Höxter und der Weg vom Rhein bei Köln nach Norddeutschland und zur Ostsee; sie kreuzten sich an der Stelle des späteren Dortmund. Den Weg von Köln benutzte Karl 775 bei seinem zweiten - dem ersten großen - Sachsenfeldzug; den Hellweg sicherte er durch Königshöfe, darunter Dortmund. ${ }^{70}$ Schon 939 war Dortmund laut Widukind von Corvey (2.16) eine befestigte urbs (kein bloßes

\footnotetext{
68 Für die Saisnes genauer: deren Einnahme durch Karl den genuinen, uns allein interessierenden Teil des Werkes und deren Behauptung durch Karl gegen Guitechins Söhne dessen schwache Fortsetzung beendet.

69 Der folgende Abriss der Geschichte Dortmunds im Hochmittelalter, sofern nicht anders angegeben, nach LM (wie Anm. 14) s. v. Dortmund, sowie Norbert Reimann, Das Werden der Stadt, und Thomas Schilp, Die Reichsstadt, beide in: Gustav Luntowski [et al.], Geschichte der Stadt Dortmund, Dortmund 1994, 13-66 bzw. 67-211.
}

70 LM (wie Anm. 14) s. v. Hellweg. 
oppidum!); 960 besaß es eine Königspfalz; spätestens ab 983 war es ein wichtiger Münzprägungsort; 990 erhielten die Gandersheimer Kaufleute «dieselben Rechte wie die Dortmunder (so dass letztere offenbar als beneidenswert galten); 1074 ist Dortmund neben Goslar und Engern eine der drei großen kaiserlichen Zollstätten in Norddeutschland; den Dortmunder Kaufleuten gewährte wahrscheinlich 1145 Konrad III., sonst sein Nachfolger Barbarossa Zollfreiheit an allen königlichen Zollstellen im Reich, dazu das Recht, nur in Dortmund angeklagt werden zu können; ${ }^{71} 1232$ schließlich war Dortmund freie Reichsstadt, die einzige, die es je in Westfalen geben sollte. Zudem griff seit Heinrichs des Löwen berühmtem Vertrag von 1161 mit den Gotländern der Dortmunder Handel immer weiter nach Nordosten aus, so dass z. B. 1229 im großen Handelsvertrag mit dem Fürsten von Smolensk auf gotländischer Seite die Fernhändler Ermbrecht und Albrecht ausdrücklich als Dortmunder genannt werden. ${ }^{72}$

Dass andererseits frankophone Kaufleute ständig sowohl bis nach als auch durch Dortmund kamen, zeigt schon eine Kölner erzbischöfliche Urkunde von 1103: ${ }^{73}$ die Kaufleute von Lüttich und Huy, unterstützt vom Lütticher Bischof Otbert, beschweren sich beim Erzbischof mit Erfolg darüber, dass die Stadt Köln die von altersher [!] üblichen Zölle heraufgesetzt hat, speziell die Durchgangszölle für ihre Fahrten «nach Sachsen und Dortmund», wo Dortmund also als einzige Stadt namentlich genannt wird; sie verkaufen, von Westen kommend, unter anderem Zinn, Tuche und Salben und holen aus Sachsen Kupfer, anscheinend auch Silber und Vieh, aus Dortmund vermutlich auch schon Waren aus dem Ostseegebiet. Wenn also in französischen Augen Guitechin einer Hauptstadt bedurfte, so hatte Dortmund seit spätestens 1100 schon aus den genannten Gründen die besten Chancen, in diese Rolle einzutreten.

\section{b Formal}

Ein bisher unerkanntes Indiz der Beziehungen zwischen dem frankophonen Raum und Dortmund steckt in der seit 1152/1153 festen Latinisierung Tremonia. Der alte Name $T(h) r o / u t m a n n i(a)$ wird bis gegen $1100 \mathrm{im}$ Wesentlichen weiter

71 Vgl. MGH DD Otto III. Nr. 66 a. 990 und Nr. 357 a.1000; DD Heinrich IV. Nr. 267 a. 1074; DD Konrad III. Nr. *134 a. 1145.

72 Hansisches Urkundenbuch, bearb. v. Konstantin Höhlbaum, 11 Bde., Halle 1876-1916, 1.7279 Nr. 232.

73 Hansisches Urkundenbuch (wie Anm. 72), 3.385-388 Nr. 601, danach Regest bei Richard Knipping, Die Regesten der Erzbischöfe von Köln im Mittelalter, II, 1100-1205, Bonn 1901, Nr. 28. 
tradiert. ${ }^{74}$ Doch tritt, wie zu erwarten, neben $T(h)$ - seit 966 zunehmend $D$ - auf; ${ }^{75}$ neben -nn- erscheint seit 997 auch - $n d$-; ferner ist das - $a$ - des mindertonigen zweiten Namensteils seit 997 häufiger zu -o-/-u- verdumpft, Letzteres z. B. in Originalurkunden Heinrichs IV.: Nr. 203 a.1068 noch Drotmanni (korrigiert aus Trotmanni), aber schon Nr.178 a.1066 Trutmunde, Nr. 267 a. 1074 Drutmunne. Und schließlich wird (auf der Basis des gesprochenen /-moni/) ein Verschriftungstyp -monia sichtbar, zunächst fragmentarisch auf Münzen der Jahre 10021024 als Trvt[m]onia, Throt[m]o[ni]a, ${ }^{76}$ dann in einer Urkunde des Erzbischofs von Köln von a. 1075 als Trutmonia, ${ }^{77}$ und in einer (nur in Abschrift des 17. Jh. erhaltenen) Urkunde Konrads III. Nr. 117 a. 1144 als Trudinonia, verschrieben aus Trudmonia. So weit die (bis wohl auf das lat. - a) unbestritten innerdt. Entwicklung des Namens. ${ }^{78}$

Schon in der nur abschriftlich (aus dem 18. Jh.) erhaltenen Urkunde Barbarossas Nr. 7 von Ende April 1152 heißt dann ein Zeuge Philippus de Tremonia, und von dem doppelten Originaldiplom Barbarossas Nr. 59/60 a. 1153 für Erzbischof Arnold von Köln an herrscht völlig fest die lat. Form Tremonia. ${ }^{79}$ Sprunghaft neu ist daran also nur das Tre-. Die regionale Forschung erkannte vor etwa zwei Jahrzehnten diese Neuerung zu Recht als erklärungsbedürftig - und wurde verständlicherweise zu Vermutungen angeregt: in den Namen könnte tres moenia hineingelesen, die ganze Form von Friedrich sozusagen aufoktroyiert sein, weil er vielleicht Dortmund zu seinem Hauptstützpunkt in Westfalen machen wollte und dazu beim Namen Einheitlichkeit um der Rechtssicherheit willen

74 Ich stütze mich auf die Belegsammlung von Derks, die in gedrängter Form Udolph bietet; vgl. Jürgen Udolph, Dortmund. Neues zu einem alten Namen, in: Beiträge zur Geschichte Dortmunds und der Grafschaft Mark 100/101 (2009/2010) 9-40, hier $10 \mathrm{f}$. Doch die kaiserlichen und erzbischöflichen Urkunden - die z.T. dort fehlen - entnehme ich den Primärveröffentlichungen.

75 Der Wandel $<$ th $>/ \theta />/ \delta /><\mathrm{d}>/ \mathrm{d} /$ ist gesamtdt., im Niederdt. fällt er hauptsächlich erst ins 11. Jh.

76 Solche mehr oder minder willkürliche Fragmentarisierung von Namen ist auf Münzen durchaus gängig, wie ein einfacher Blick in irgendein Werk zur Numismatik lehrt.

77 Knipping (wie Anm. 73), Nr. 1054.

78 Was die schwierige Frage der Etymologie des Namens angeht, scheint Udolphs (wie Anm. 74) germ. throt 〈Kehle〉 (hier 〈Einschnitt〉) $*^{\star}$-mund 〈Anhöhe, Hügel〉 der bisher einzige akzeptable Vorschlag. Ob -nd- oder -nn- als primär zu gelten hat, ist für die Frage des frz. Einflusses auf die spätere Form Tremonia irrelevant, da /ndǐ/ im Frz. mit altem /nǐ/ in /ñ/ zusammenfällt, vgl. Burgundia $>$ Bourgogne, verecundia $>$ vergogne ganz wie Bononia $>$ Boulogne, vlat. *caronea $>$ *caronia $>$ charogne.

79 Man darf sagen: bis heute; denn die lat. Form ist in der Stadt allgemein bekannt geblieben (bis 1945 Name einer dortigen Tageszeitung; noch heute Tremonia-Park an der Stelle der einstigen Zeche Tremonia sowie Clubs und Firmen dieses Namens). 
brauchte ... ${ }^{80}$ Aber dass Dortmund schon um 1150 eine Drei-Mauern-Stadt war oder dass es $\mathrm{zu}$ irgend einer Zeit in irgend einer distinktiven Weise als solche empfunden wurde, ist durch kein Dokument belegt; bei Hineinlesen eines lat. Sinnes wäre nicht -monia, sondern eben -moenia bzw. -menia zu erwarten; dass Friedrich Dortmund eine solche Rolle zugedacht hätte, ist nur aus der Urkunde selbst herausgelesen und wird durch die Folgeereignisse nicht bestätigt, so dass hier ein Zirkelschluss droht; dass schließlich um diese frühe Zeit die kaiserliche Kanzlei für irgend einen der Hunderte Ortsnamen von Mittelitalien bis Holstein, die jährlich in ihre Urkunden eingingen, außer bei der Übernahme antiker Namen Schreibungskonstanz angestrebt hätte, ist leicht zu widerlegen. ${ }^{81}$

Dem Romanisten drängt sich eine andere Lösung auf. Die doppelte Originalurkunde von 1153 ist laut MGH-Edition in beiden Ausfertigungen EmpfängerUrkunde, außerhalb der kaiserlichen Kanzlei von Kölner erzbischöflichen Schreibern formuliert und geschrieben. Es ist auffällig, dass in Köln fast gleichzeitig auch außerhalb der erzbischöflichen Kanzlei für «Dortmund» schon Tremonia in Gebrauch war: zwischen 1164 und 1167 benutzte der Klostercustos Theodericus von Sankt-Heribert zu Deutz in seiner erhaltenen Originalhandschrift eben diese Form: in Tremonia; unmittelbar vorher hat er von talenta Tremonensis monetae gesprochen. ${ }^{82}$ Den Ton scheint also nicht die kaiserliche Kanzlei, sondern die des Erzbischofs (und zugleich Bischofs) angegeben zu haben, die für die lat. Formen der Ortsnamen ihres Sprengels eher als autoritativ gelten musste und der die kaiserliche in diesem Fall folgte. Wie die kaiserliche, so blieb auch die Kölner Kanzlei in der Folge bei Tremonia. ${ }^{83}$

Nun waren in Köln Einflüsse seines frankophonen Suffraganbistums Lüttich früh zu spüren; zudem hatte es immer individuelle Kontakte mit dem frz. Sprachgebiet gegeben wie etwa, dass Erzbischof (Sankt) Heribert (999-1021), der Gründer des Klosters Deutz, in Metz und Gorze gebildet war. Doch seit dem Ersten Kreuzzug hatte sich Köln frz. Einflüssen genereller geöffnet: Erzbischof Friedrich I. (1100-1135) hatte in Frankreich studiert und blieb so frankophil,

80 So Reimann (wie Anm. 69) 46.

81 Noch in Barbarossas Originalurkunden aus den Jahren 1158-1190 (MGH DD Friedrich I., Bd. 2-4) findet man nebeneinander Magantia/Magontia/Maguntia/Mogontia samt Magontinus/ Maguntinus/Mogontinus/Moguntinus, Ratisbona/-pona samt Radis-/Rathisponensis, Salce-/Saltz-/ Salz-/Salze-/Salzi-burch/-burg/-purg samt -ensis etc. Das gilt auch für Orte, deren Aufstieg Barbarossa persönlich am Herzen lag, so in dem geplanten zweiten, aber nicht zustande gekommenen ostfränkisch-egerländisch-vogtländischen Herzogtum der Staufer die junge Stadt Nǒremberg/Nuerenberc/Nuremberg/Nurenberc/Nurenberg/Nurinbergo samt Norumbergensis.

82 MGH SS. 14.564 bzw. 563.

83 Z. B. a. 1179 und 1189, Knipping (wie Anm. 73) Nr. 1136 und 1336. 
dass er seine Nichten mit den Grafen von Champagne und von Nevers verheiratete und bei der Königswahl von 1125 weder für den Staufer noch für den Sachsen, sondern für Karl von Flandern, Lehnsmann des französischen Königs, eintrat. ${ }^{84}$ Ihm folgte auf dem Erzbischofsstuhl sein Agnat Bruno II. (1131-1137), später sein Großneffe Friedrich II. (1156-1158). Auch Sankt-Heribert stand da nicht abseits: Erzbischof Friedrich I. war befreundet mit dem bedeutenden Theologen, der als Rupert von Deutz in die Geschichte eingegangen ist, aber gebürtiger Lütticher, also Frankophone, war, erst im Alter von über 35 Jahren nach Deutschland kam und von 1120-1129/30 als Abt von Sankt-Heribert wirkte. Das alles macht einen frz. Einfluss auch auf die erzbischöfliche Kanzlei und bis nach Sankt-Heribert plausibel.

Akzeptiert man diese Voraussetzung, so ist die Schlussfolgerung einfach. Im Afrz. wird germ. $/ \Theta />/ \mathrm{t} /$, nicht $/ \mathrm{d} / ;^{85}$-tm- wird früh $\mathrm{zu}-\mathrm{m}^{-86}$ (und war im genuinen Lat. unbekannt, musste also auch im Mlat. eher gemieden werden); vortoniges $-o$ - > - $e$ - ist schon im 12. Jh. ausgesprochen häufig, vielleicht sogar lautgesetzlich, sowohl vor Nasal als auch dissimilatorisch vor -ó- und natürlich beim Zusammenfall beider Bedingungen. ${ }^{87}$ Folglich war afrz. Tremo(i)gne / tremọño/ < Throtmonnia die zu erwartende Entwicklung, die dann auf mlat. Tremonia führte.

Lässt sich auch das Kommen dieser Form aus der Frankophonie nach Köln dokumentieren? Wenigstens exemplarisch kann dazu, ein halbes Jahrhundert vor der Barbarossa-Urkunde, die oben erwähnte Kölner Urkunde von 1103 für die wallonischen Kaufleute dienen; denn auch sie gibt sich als EmpfängerUrkunde $\mathrm{zu}$ erkennen, ${ }^{88}$ und «nach Dortmund heißt darin versus Tremonge,

84 LM (wie Anm. 14) s. v. Friedrich 44.

85 Ich zitiere hier und in den beiden folgenden Anm. ausschließlich afrz. Formen des 12. Jh.: Theud-rîk > afrz. Tierri, heute rein graphisch Thierry, und andere Theud-Namen; theodisk > afrz. tiedeis $>t$ (h)iois; throp $>$ afrz. trop.

86 Rimer (wenn es ganz oder hauptsächlich zu rhythmus gehört) Ph. de Thaon Comp.; rime Chrét.; seme (< septimum) Chrét.; oime (< ${ }^{\star}$ octimum statt octavum) Beneeit $C D N$, uime Eneas, Alexandre.

87 Demenie/demeine/demaine (< domini[c?]um) Rol., Ch. de Guill., Chrét.; trench(i)er ( $<$ truncare) Rol., Cour. Louis; Rencesvals (< Roncesvals < Rozaballes x ronces < rumices) Rol.; nen (< non) Alexius, Rol., Cambr.Ps., Chrét.; nenil (< non illud) Cour. Louis, Ch. de Guill., Chrét.; reont, reoignier (< rotundum, *rotundiare) Chrét.; seror (< soror), serorge (< sororius) Chrét.; secorre (< succurrere) Wace, Eneas, Ch. de Guill., Chrét.; secors (< succursum) Chrét.; sejorner (< ${ }^{\star}$ subdiurnare) Cour. Louis, Ch. de Guill., Chrét.; sejor(n) Beneeit CDN, Chrét.; enor (< honor) Charroi, Rou, Chrét.; semondre (< submonere) Chrét.

88 S. oben Anm. 73. Die Urkunde ist zwar nur in einer Kopie des 17. Jh. erhalten, aber einer notariellen (Lüttich UB, Ms. 251, f. 149). 
schon mit dem charakteristischen Tre- $;^{89}<\mathrm{ng}>$ ist in dieser Frühzeit noch eine akzeptable Graphie auch für / $\tilde{n} / .^{90}$

In der Tat erscheint denn auch Tremoigne (jetzt mit dem im Afrz. üblichen

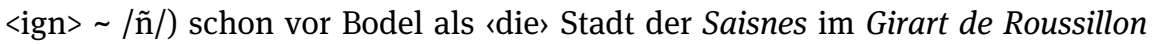
v. 2774, und zumindest etwa gleichaltrig mit Bodel ist der Renaut de Montauban mitsamt seiner historisch noch immer nicht sicher erklärten Rolle von Tremoigne speziell im Schlussteil (v. 6992 und 12215-14279 ed. Thomas).

Der Fall kann ein gewisses allgemeineres Interesse beanspruchen: er zeigt nicht nur, wie zu erwarten, dass die Geistlichkeit, weil Hauptträger und Haupttraditor der mlat. Schriftlichkeit, auch als Autorität für diese galt; sondern er lässt zudem vermuten, dass selbst eine rom. Volkssprache, weil erkennbar dem Lat. nahe, unterschwellig an diesem Prestige teilhaben konnte.

\section{Der narrative Kern}

Doch gab es für den Aufstieg Dortmunds zur Hauptstadt Guitechins einen noch wichtigeren Grund als seine wirtschaftliche Bedeutung und seine engen Beziehungen nach Westen: es lag bei der Hohensyburg 〈hinter〉 der Ruhr. Damit sind wir beim narrativen Kern der Sachsenepik: er ist historisch, stammt aus Karls zweitem Sachsenkrieg, dem Feldzug von 775.

Auf seinen historischen Gehalt befragt, beeindruckt das Rolandslied dadurch, dass nicht nur die Vernichtung von Karls gesamter Nachhut durch einen Überraschungsangriff eben eine solche geblieben ist, ${ }^{91}$ sondern dass auch der Ort der Handlung essenziell derselbe geblieben ist. Welcher Franzose des Mittelalters, der den Weg über den Pass von Roncevaux nahm, wird nicht für einen Augenblick stehen geblieben sein im Gedenken: «hier also war es` - ? Besitzt die Sachsenepik auch ein solches Kernszenario? Selbst vor Bédier hätte die Forschermehrheit die Frage verneint; die dreiseitige Miszelle, in der 1893 Schultz [-Gora] ${ }^{92}$ sie bejahte, machte schon deshalb wenig Eindruck, weil der Autor darin hauptsächlich von der Bedeutung des Namens Rune sprach und

89 Das Ms. hat wohlgemerkt Tremonge; vgl. den Apparat im Hansischen Urkundenbuch. Leider hat Höhlbaum als dessen Editor geglaubt, Tremonge zu Tremunge semendieren` zu sollen. Knipping hat dann in sein Regest kommentarlos nur Tremunge übernommen.

90 Pope, Mildred, From Latin to Modern French with Especial Consideration of AngloNorman, ${ }^{2}$ Manchester 1952, § $695 \mathrm{f}$.

91 Wenn ich hier meiner eigenen Überzeugung Ausdruck verliehe, würde ich auch Roland als militärischen Anführer dieser Nachhut und einiges andere als Konstanten von 778 bis nach 1100 nennen, doch verlangt die obige Aussage einen Minimalkonsens.

92 Oscar Schultz[-Gora] (wie Anm. 61), passim. 
selbst dabei, wie wir oben festgestellt haben, einen richtigen Kern glaubte nebulös abzusichern zu sollen. Heute sind Schultz' Zeilen so vergessen, dass z. B. Paul Aebischer sie nicht kannte, als er in einem 16-seitigen Aufsatz zu dem Fazit kam, ${ }^{93}$ dass in der Sachsenepik außer den beiden Namen des Siegers Charlemagne und des Besiegten Widukind sowie dem vagen Wissen, dass dieser Krieg irgendwo im Nordosten stattfand, nichts historisch sei; ebensowenig weiß Annette Brasseur ${ }^{94}$ etwas von Schultz.

Aber Schultz hatte Recht! Allerdings: um diesen Kern der Sachsenepik würdigen zu können, müssen wir etwas weiter ausholen, als Schultz das tat. Karl begann (nach den Reichsannalen) seinen ersten Sachsenfeldzug 772 von Worms aus; er zog nordwärts durch das frankisierte Hessen und nahm (cepit) die sächsische Eresburg im heutigen Ober-Marsberg (Hochsauerlandkreis), zerstörte dann die nahe Irminsul. Die Eresburg ist heute von der westfälisch-hessischen (damals also sächsisch-fränkischen) Landesgrenze nur $3 \mathrm{~km}$ entfernt, und es gibt keinen Grund zu der Annahme, dass es damals wesentlich mehr gewesen sein könnten. Dann zog Karl zu einem ungenannten Ort an der Weser, erhielt zwölf Geiseln und kehrte in Franciam zurück. Von der Eresburg beträgt die Entfernung zu den Weserübergängen von Höxter-Braunsberg und Herstelle, die Karl später nachweislich benutzte, 69 bzw. 61 Straßenkilometer; das sind zwei bis drei Tagesmärsche. Von Höxter zurück zur hessischen Grenze bei Karlshafen sind es 24, von Herstelle sogar nur 4 km. Verglichen mit Karls späteren Feldzügen, kann man also den von 772 fast einen Spaziergang nennen; es ist nicht erstaunlich, dass er in der Epik keine Spuren hinterlassen hat.

Während Karl 773/774 das Langobardenreich vernichtete, unternahmen die Sachsen einen Rachezug ins Hessische, wo sie Fritzlar brandschatzten, aber spontan wieder abzogen. Bei der Rückkehr aus Italien 774 schickte ihnen Karl in Eile noch vier Scharen nach, von denen laut den Reichsannalen drei in für sie siegreiche Gefechte verwickelt wurden, ohne dass wir Näheres erfahren. Das und die Tatsache, dass der König nicht mitzog, deuten auf eine improvisierte Minimalmaßnahme.

In gewissem Sinne waren somit erst die Ereignisse von 775 der wirkliche premier choc. Nach den Reichsannalen sammelte Karl sein Heer diesmal bei Düren und zog - cum totis regni viribus, wie die Annales qui dicuntur Einhardi präzisieren $^{95}$ - zur Hohensyburg, Sigiburgum castrum. Ein Blick auf die Karte

93 Paul Aebischer, Lélément historique dans les chansons de geste ayant la guerre de Saxe pour thème, in: P. Ae., Des annales carolingiennes à Doon de Mayence, Genève 1975, 223-239, hier $236 \mathrm{f}$.

94 Wie Anm. 1 und 10.

95 Annales Regni Francorum inde ab a. 741 usque ad a. 829, qui dicuntur Annales Laurissenses Maiores et Einhardi, ed. Fridericus Kunze, Hannover 1895 (MGH SS.schol. 6), p. 41. 
zeigt eindeutig, dass er dazu den Rhein von Köln, dem Couloigne der Saisnes, nach Köln-Deutz, dem Saint Herbert dou Rin der Saisnes, überquerte. Etwa $55 \mathrm{~km}$ weiter verlief die westfälische (damals also sächsische) Westgrenze östlich des heutigen Wuppertal. ${ }^{96}$ Doch ergab sich eine natürliche, aber sogleich imposante Verteidigungslinie erst abermals $15 \mathrm{~km}$ weiter, wo die Straße jenseits von Hagen (heute als L 704), nunmehr mit südnördlichem Verlauf, die Ruhr dicht unterhalb der Lenne-Mündung überquerte und unmittelbar danach zur Hohensyburg hochstieg. Es passt also durchaus, wenn bei Bodel die Franken nicht lange nach dem Aufbruch von Köln-Deutz schon in Feindesland plündern können (v. $1271 \mathrm{AR} \sim 1207 \mathrm{LT}$ ) und erst nach einem weiteren kurzen Ritt a esperon brochant die Rune zu Gesicht bekommen (v. 1273 AR 1209 LT).

Die sich 775 ergebende Situation kann man gut im Internet-Atlas $w w w$. maps.google.de überblicken. Dicht an das nördliche Ruhrufer schließt dort die Bezeichnung «Ruhrsteilhänge Hohensyburg» an; auf dem nur von Nordosten zugänglichen Plateau oberhalb dieser Steilhänge - durchschnittlich mehr als 100, maximal mehr als 130 m über dem Fluss - befinden sich die Ruinen der großen sächsischen Wallburg, die mit bis zu $700 \times 310 \mathrm{~m}$ den Großteil des Plateaus in Anspruch nahm und sich bis unmittelbar an die Steilwand erstreckte. ${ }^{97}$ Die Straße muss also an der Westflanke des Plateaus um fast $100 \mathrm{~m}$ steigen; heute überquert sie dazu, um ihren Steigungswinkel auf moderne Verhältnisse zu drücken, kurz nach dem Ruhrufer in einer vollen Schleife ansteigend auf einer Talbrücke sich selbst, hat aber auch danach bei ständiger Steigung noch zwei Kurven von je $180^{\circ}$ vor sich, bevor von ihr, spitzwinklig-zurück, die heutige Seitenstraße zum Burg-Plateau abzweigen und sie selbst ungehindert weiter die verbleibenden $13 \mathrm{~km}$ nach Dortmund-Mitte verlaufen kann; der alte Straßenverlauf kann nicht wesentlich anders, nur steiler gewesen sein. ${ }^{98}$ Von der Wallburg blickt man weit in die offene Tallandschaft hinaus, konnte also einen Feind früh ausmachen; vom Flussufer aus - oder besser noch von einer etwas erhöhten Position auf der Straße - konnte man ihm den Flussübergang durch einen Pfeilregen erschweren, am Flussufer ihm die von da aufsteigende Straße versperren, und selbst falls er dort durchdrang, musste für ihn der gesamte

96 Die naturgeographische Grenze verläuft etwa $6 \mathrm{~km}$ östlich der Wuppertaler Stadtgrenze auf dem Nord-Süd-Höhenzug vor dem Ennepetal; aber dieser senkt sich nach Westen flach, nach Osten steiler, so dass er als Verteidigungslinie der Sachsen unbrauchbar war.

97 Ausführlich dazu Philipp Hömberg, Die Hohensyburg, Münster 2000, passim, und ders., Burgen des frühen Mittelalters in Westfalen, in: Hinter Schloss und Riegel. Burgen und Befestigungen in Westfalen, hg. vom Landschaftsverband Westfalen-Lippe, Dortmund 1997, 120-159. 98 Laut Hömberg (2000, wie Anm. 97), 3, gab es vom Fluss zum Plateau seit 1903 bis zum Abbau 1923 sogar eine Zahnradbahn für den Tourismus! 
Aufstieg verlustreich werden, wenn er ständig aus erhöhten Positionen von vorn oder in der Flanke angegriffen wurde. ${ }^{99}$

Karl hat damals dennoch das Sigiburgum castrum eingenommen, nach den Annales qui dicuntur Einhardi sogar primo impetu [...] pugnando - vermutlich, indem er das Hindernis wie bei seinem berühmten Alpenübergang knapp zwei Jahre vorher auf einem weiten Umweg umging ${ }^{100}$ und von Nordosten eindrang.

Auf dem Feldzug von 775 erzwang Karl nach der Eroberung der Hohensyburg noch den Weser-Übergang und erreichte die Oker; dort, nur schätzungs-

99 Einen kompletten Meinungswandel bezüglich des Geländes um die Hohensyburg hat innerhalb eines Jahres Annette Brasseur durchgemacht. Im Index ihrer Ausgabe (1989, wie Anm. 1) hieß es noch untertreibend, Tremoigne sei probablement Dortmund, und die Rune sei gar eine rivière d'identification difficile qui sépare le territoire de Charlemagne de celui des Saxons, was im ersten Teil des Satzes ein bequemer Agnostizismus, im zweiten Teil sogar, wie oben gezeigt, falsch ist. In der begleitenden Monographie (1990, wie Anm. 10, 273 s.) ist Brasseur dann nach dem Studium einer nicht sehr detaillierten Karte zu dem anderen Extrem gelangt, der Meinung nämlich, Bodel müsse das Ruhrtal bei Hohensyburg persönlich gekannt haben. Kaum! Denn in diesem Fall hätte er die Roche au Jaiant nicht auf Karls Seite des Flusses gesetzt, wo in der Realität kilometerweit nur nahezu ebenes Land zu sehen ist, und hätte den dort mündenden kleinen Fluss nicht willkürlich Tarsie (v. 2173 A), Carsie (v. 2173 R, 1929 L) oder Garsie (v. 1929 T) genannt, sondern an die realen Namen Lenne oder Volme angepasst; so gut wie sicher fiktiv ist an der Rune auch die etwa ein Dutzendmal genannte Furt von Mor(r)estier/Mor(t)itier/Montestier (zu afrz. estier 〈canal〉 < lat. aestuarium?), ebenso in Bodels Deutschlandbild ein Gewässer Maisence/Maissance o. ä. dicht westlich von Aachen (oder soll das ein Diminutiv von Maas/Muese suggerieren?) sowie der oder die Orte Hauteme (kaum Haltern, das erst 1289 Stadt- und damit Befestigungsrechte erhielt) und/oder au Glore/Rogles in Rheinnähe. Von deutschen Ortschaften kennt er außer Aachen, Köln, Deutz und Dortmund anscheinend nur Goslar (Colaire v. 165, 181 ART Golane v. 162, 179 L, beide leicht verderbt aus ${ }^{\star} G o$ (s)laire < Goslaria, der normalen Latinisierung), das im 11. und 12. Jh. etwa 20-mal eine Reichsversammlung beherbergt hatte, aber vor allem seit etwa 900 Zentrum jenes Silber- und Kupferabbaus war, der bald die größte Einnahmequelle der Kaiser wurde, dann zur Verfeindung Barbarossas mit Heinrich dem Löwen führte und schon deshalb auch in Frankreich bekannt sein musste; eine andere Frage ist allerdings, ob sich Bodel die Lage von Goslar innerhalb Sachsens einigermaßen richtig vorstellt. Insgesamt hat er eine erstaunlich geringe Eigenkenntnis von Deutschland. Umso zuversichtlicher darf man die richtige Grundkonstellation um den Komplex Rune-Tremoigne einer Vorstufe Bodels zurechnen. - Die gerade erwähnte Identifizierung von Colaire mit Goslar ist das einzige Detail, in dem ich mich in Übereinstimmung befinde mit Franz Settegast, Die Sachsenkriege der französischen Volksepos auf ihre geschichtlichen Quellen untersucht, Leipzig 1908, hier 50 mit Anm. 1. Settegast stellt die afrz. Sachsenepik dar als ein schwindelerregendes Kaleidoskop von Einflüssen: von den Feldzügen des Germanicus, Vitellius und Julian über Kriege der Thüringer des 6. Jh. und der Ostgoten bis zur Person des Großfürsten Jaroslav von Kiev (†1054); insgesamt wirkt die Schrift wie eine karikaturhafte Übertreibung alles dessen, wogegen Bédier zu Felde zog.

100 So wie heute die Autobahn (A1 = E 37 nordostwärts bis Westhofener Kreuz, dann A 45 = E 41 nordwestwärts). 
weise $70 \mathrm{~km}$ vor der damaligen Slavengrenze, stellten ihm die Ostsachsen Geiseln und schwuren den Untertaneneid. Auf Karls Rückweg taten dies bei Bückeburg ebenso die mittleren Sachsen (Engern), kurz darauf die Westfalen. Am Ende des Feldzugs von 775 konnte man also in einem ganz anderen Maß als 772 glauben, Karl habe damit den Sachsenkrieg siegreich beendet.

Die Wallburg Hohensyburg scheint in der Folge noch einige Zeit von einer Truppe belegt gewesen zu sein; doch lag sie seit langem in Ruinen, als zwischen 1090 und 1100 ein Mönch aus der Osnabrücker Gegend die Sigeburg [...] super Ruram aufzählte unter den drei ihm imponierenden heidnisch-sächsischen castella, quae huc usque destructa videmus ${ }^{101}$ Seine Worte zeigen nicht nur, wie eindrucksvoll die Ruinen der Wallburg waren, sondern auch, dass man als eine Selbstverständlichkeit ihren heidnischen Ursprung kannte - was automatisch einschloss, dass Karl der Große, da er ja das ganze Sachsenland erobert hatte, auch diese Burg erobert hatte.

Gerade hierin liegt aus der Sicht unseres Themas ein entscheidender Vorzug der Hohensyburg z. B. gegenüber der hochsauerländischen Eresburg, aber auch gegenüber allen Schlachtorten weiter im Norden: während nur verschwindend wenige frankophone Kaufleute je ins Hochsauerland - und relativ wenige nach Norddeutschland - gekommen sein werden, war die Route Köln-HohensyburgDortmund 1103 schon «seit altersher〉 zumindest für die Wallonen, wie oben gezeigt, ein vielbegangener Handelsweg. ${ }^{102}$ Wenn sie bei jedem ihrer Züge mitsamt ihren Ladungen das Plateau mit den Ruinen der Volksburg ersteigen mussten, konnten die Einheimischen es ihnen jederzeit bestätigen: «hier hat einst Karl der Große die Sachsen geschlagen.> Damit kommen wir zu unserem wichtigsten Schluss: wenn somit die Eresburg einerseits, Norddeutschland andererseits für die afrz. Erinnerung ausfielen, wurde die Schlacht von 775 an der Ruhr eben zum Inbegriff des Sachsenkrieges überhaupt, und da Karls Sachsenkriege, wie jedermann wusste, 〈lange〉 gedauert hatten, dauerte nun die Schlacht an der Ruhr 〈lange», und Dortmund rückte - unhistorisch, aber nicht historisch unbegründet - zur narrativ unentbehrlichen Hauptstadt Widukinds oder vielmehr Guitechins gleich hinter der Ruhr auf.

\section{Schlussfolgerungen}

Wir können unsere Ergebnisse nun auch zeitlich schichten. Der historische Karl hatte nie Probleme mit dem Überschreiten des Rheins, wohl aber in einem für

101 Vita Bennonis II (MGH SS.schol. 56.15); zum Datum dort p. V.

102 Wie ein Blick auf die Karte lehrt, kann sie das ebenso - wenn auch wohl in geringerem Umfang als für Wallonen - für Kaufleute aus dem weitaus größeren Teil der Nordhälfte der 
seine Sachsenkriege exemplarischen Fall mit dem Überschreiten der Ruhr; zudem passt Dortmund, das in allen drei Fassungen vorkommt, besser zur Rune als zum Rin. Dadurch kommt dem Saisnes-Erzähltyp ein entscheidender Vorsprung an Historizität und in diesem Fall auch die zeitliche Priorität zu. Denn der Rhein (in KMS I und V) ist demgegenüber (graphisch-phonisch, aber mehr noch narrativ) lectio facilior, einleuchtend erst infolge der politischen Umwälzungen des 10./11. Jh. durch die Rheingrenze als Frankreichs nunmehrige Irredenta. Für die Verselbständigung des Rhein-Erzählstrangs ist von vornherein 1100 terminus ante quem, schon weil er vor dem PT (Kap. 33) voll entwickelt gewesen sein muss. Aber der Rhein ist lang; so tritt er uns in zwei Lokalisierungsformen entgegen. Zum einen hatte man die Erfahrungen, die Karl der Einfältige 920 bei Worms gemacht hatte, im Laufe des 11. Jh. (eher wohl früh) auf Karl den Großen um-erinnert und damit in das Sachsenkriegs-Thema integriert (was sich in der KMS V spiegelt). Zum anderen wusste niederrheinischer Regionalstolz noch, dass Karl zum Sachsenkrieg den Rhein «in der Regel〉 bei Wesel überschritten hatte, und konnte dieses Wissen (zusammen mit der niederrheinischen Form der Schwanenritter-Sage) dem Lütticher Kompilator der Vie de Charlemagne vermitteln (die sich in der KMS I spiegelt). Älter als diese geographischen Spaltungen ist das Motiv des Schlussduells, älter muss auch die Integration des Themas «Karl baut (mit Hilfe aller seiner Völkerschaften) eine Brücke> in den Sachsen-Kontext sein. Doch ist selbst damit noch keine volle mündliche Kontinuität des Sachsenkrieg-Erzählstoffes im Sinne Menéndez Pidals, also von den Ereignissen an, garantiert; denn man kann nicht ausschließen, dass sich das Wissen um den Kampf an der Hohensyburg erst im Anblick der Ruinen (wieder)entzündet hat. Überhaupt bleibt im starken Anteil der Geographie an den Erklärungen Bédiersches Erbe präsent. Für die volle Kontinuität à la Menéndez Pidal kann man als Argument von einiger Wahrscheinlichkeit nur die Ähnlichkeit des merowingischen Erzählmoduls (Konfrontation der Franken mit den Sachsen über den Fluss mit Rufen und tollkühnen Einzelüberquerungen) geltend machen, wobei allerdings die Kraft des Argumentes beträchtlich gestärkt wird durch die Unveränderlichkeit der Geographie und damit der Probleme, die sie den Franken bei jedem neuen Sachsenzug verursachte. Eben: Epik um einen Fluss.

Galloromania, nämlich aus einem Großteil der Pikardie ebenso wie aus Paris und der Champagne, gewesen sein. 


\section{Postskriptum 2018}

Erun(n)ia neben Irun(n)ia. Nachträglich sehe ich, dass für Iruña um 1100 auch Erun(n)ia gängig ist - so in den Urkunden der Könige Sancho Ramírez von 1085, Pedro I. von 1097 und 1101 sowie Alfonso el Batallador von 1116, zitiert z. B. bei Manuel Álvar, Estudios sobre el dialecto aragonés, Bd. I, Zaragoza 1973, 23, 25, 55. Diese Namensform vereinfacht (und stärkt damit) die obige Ableitung el río de_Iruña bzw. jetzt de_Eruña $>$ der Fluss Rune. 
